\title{
The Weibull Topp-Leone Generated Family of Distributions: Statistical Properties and Applications
}

\author{
Hamid Karamikabir ${ }^{1}$, Mahmoud Afshari ${ }^{1}$, Haitham M. Yousof ${ }^{2}$, Morad Alizadeh \\ 1 , and Gholamhossein G. Hamedani ${ }^{3}$ \\ ${ }^{1}$ Department of Statistics, Persian Gulf University, Bushehr, Iran. \\ ${ }^{2}$ Department of Statistics, Mathematics and Insurance, Benha University, Benha, Egypt. \\ ${ }^{3}$ Department of Mathematics, Statistics and Computer Science, Marquette University, USA.
}

Received: 02/10/2018, Revision received: 03/06/2019, Published online: 05/06/2019

\begin{abstract}
Statistical distributions are very useful in describing and predicting real world phenomena. Consequently, the choice of the most suitable statistical distribution for modeling given data is very important. In this paper, we propose a new class of lifetime distributions called the Weibull Topp-Leone Generated (WTLG) family. The proposed family is constructed via compounding the Weibull and the Topp-Leone distributions. It can provide better fits and is very flexible in comparison with the various known lifetime distributions. Several general statistical properties of the WTLG family are studied in details including density and hazard shapes, limit behavior, mixture representation, skewness and kurtosis, moments, moment generating function, incomplete moment. Different methods have been used to estimate its parameters. The performances of the estimators are numerically investigated. We have discussed inference on the new family based on the likelihood ratio statistics for testing some
\end{abstract}

Hamid Karamikabir (h_karamikabir@yahoo.com)

Corresponding Author: Mahmoud Afshari (afshar@pgu.ac.ir)

Haitham M. Yousof (haitham.yousof@fcom.bu.edu.eg)

Morad Alizadeh (m.alizadeh@pgu.ac.ir)

Gholamhossein G. Hamedani (g.hamedani@mu.edu) 
lifetime distributions. We assess the performance of the maximum likelihood estimators in terms of the biases and mean squared errors by means of a simulation study. The importance and flexibility of the new family are illustrated by means of two applications to real data sets.

Keywords. Generating Function, Lifetime Distributions, Maximum Likelihood Estimation, Quantile Function, Topp-Leone Distribution, Weibull Distribution.

MSC: 60E05; 62F10; 62G05.

\section{Introduction}

Although many distributions have been developed, there are always rooms for developing distributions which are either more flexible or better fit for specific real world scenarios. This has motivated researchers in seeking and developing new and more flexible distributions. The statistical literature contains many new classes of distributions which have been constructed by extending known families of continuous distributions providing more flexibility via adding one or more parameters to the baseline model. These new families have been used for modeling data in many applied areas such as engineering, economics, biological studies, and environmental sciences. Gupta et al. (1998) defined the exponentiated-G (Exp-G) class which consists of raising the cumulative distribution function (cdf ) to a positive power parameter and proposed the exponentiated exponential (EE) distribution with the cdf (for $x \geq 0$ ) $F(x)=[1-\exp (-\lambda x)]^{\alpha}$, where $\lambda, \alpha>0$. This cdf is simply the $\alpha$ th power of the standard exponential cumulative distribution function. Further details were explored by Gupta, and Kundu (2001).

The generalized distributions were pioneered by Marshall and Olkin (1997), Eugene et al. (2002), Cordeiro et al. (2013), Alzaatreh et al. (2013), Yousof et al. (2015, 2016, 2018), Brito et al. (2017), Alizadeh et al. (2017, 2018), Nofal et al. (2017), Afify et al. (2017), Hamedani et al. (2018) and Korkmaz et al. (2017, 2018a, 2018b, 2018c, 2019a, 2019b), among others.

Based on T-X idea (Alzaatreh et al. (2013)), a new extended class called the The Weibull Topp-Leone Generated (WTLG) family of distributions is defined with cdf given by

$$
F(x)=1-\exp \left\{-\left(\frac{\left[1-\bar{G}(x ; \psi)^{2}\right]^{\alpha}}{1-\left[1-\bar{G}(x ; \psi)^{2}\right]^{\alpha}}\right)^{\beta}\right\}, \quad x \in \mathbb{R},
$$


where $\alpha, \beta>0$ and $\bar{G}(x ; \psi)=1-G(x ; \psi)$. Its corresponding probability density function (pdf) is

$$
\begin{aligned}
f(x)= & \frac{2 \alpha \beta g(x ; \psi) \bar{G}(x ; \psi)\left[1-\bar{G}(x ; \psi)^{2}\right]^{\alpha \beta-1}}{\left\{1-\left[1-\bar{G}(x ; \psi)^{2}\right]^{\alpha}\right\}^{\beta+1}} \\
& \times \exp \left\{-\left(\frac{\left[1-\bar{G}(x ; \psi)^{2}\right]^{\alpha}}{1-\left[1-\bar{G}(x ; \psi)^{2}\right]^{\alpha}}\right)^{\beta}\right\}, \quad x \in \mathbb{R} .
\end{aligned}
$$

The hazard rate function (hrf) $h(x)=f(x ; \psi) /[1-F(x ; \psi)]$ is

$$
h(x)=2 \alpha \beta g(x ; \psi) \bar{G}(x ; \psi)\left[1-\bar{G}(x ; \psi)^{2}\right]^{\alpha \beta-1}\left\{1-\left[1-\bar{G}(x ; \psi)^{2}\right]^{\alpha}\right\}^{-(1+\beta)} .
$$

On the other hand, we know that the cdf of the Topp-Leone distribution is given by

$$
F_{T L-G}(x)=\left[1-(1-x)^{2}\right]^{b}, \quad 0 \leq x \leq 1 .
$$

An interpretation of the WTLG family of distributions can be given, in a context similar to (Cooray (2006)) as follows: Let $Y$ be a lifetime random variable with a certain continuous distribution $G$. The odds ratio that an individual (or component) following the lifetime $Y$ will die (fail) at time $x$ is $F_{T L-G}(x) /\left(1-F_{T L-G}(x)\right)$. Consider that the variability of this odds of death is represented by the random variable $X$ and assume that it follows the Weibull model with scale 1 and shape $\beta$ (Bourguignon et al. (2014)). We can write

$$
\operatorname{Pr}(Y \leq x)=\operatorname{Pr}\left(X \leq \frac{F_{T L-G}(x)}{1-F_{T L-G}(x)}\right)=F(x ; \alpha, \beta, \psi),
$$

which is given by equation (1.1).

If $U \sim U(0,1)$, then

$$
X_{u}=Q_{G}\left\{1-\left[1-\left(\frac{[-\log (1-U)]^{\frac{1}{\beta}}}{1+[-\log (1-U)]^{\frac{1}{\beta}}}\right)^{\frac{1}{\alpha}}\right]^{\frac{1}{2}}\right\} .
$$

The justification for the practicality of WTLG family is based on the wide use of the W-G family. We are motivated to introduce the WTLG family of distributions 
since it exhibits increasing, decreasing as well as buthtab hazard rates as illustrated in Figures 2. It is shown in Subsection 3.2 that the WTLG family of distributions can be viewed as a mixture of the exponentiated-G distributions. The new family can be viewed as a suitable model for fitting the right-skewed, symmetric and bimodal data sets. The WTLG family of distributions outperform several of the well-known lifetime distributions with respect to a real data application as illustrated in Section 8.

This paper is organized as follows. Two special cases are presented in Section 2. In Section 3, some of the mathematical properties of the new family are derived.. Section 4 deals with characterization of WTLG. Maximum likelihood estimation for the model parameters under uncensored data are addressed in Section 5. Likelihood ratio test is studied in Section 6. A simulation study is performed in Sections 7 to assess the performance of the estimators. In Section 8 , the potentiality of the proposed class is illustrated by means of two real data sets. Finally, Section 9 provides some concluding remark.

\section{Special Cases}

In this section we consider two special cases of the proposed family.

\subsection{The Weibull Topp-Leone Generated Normal Family (WTLG-N)}

Taking $G(x ; \psi)$ and $g(x ; \psi)$ to be the cdf and pdf of the normal $N\left(\mu, \sigma^{2}\right)$ distribution, respectively in (1.2), where $\xi=(\mu, \sigma)^{T}$, the WTLG-N density function is given by

$$
f(x)=\frac{2 \alpha \beta \phi\left(\frac{x-\mu}{\sigma}\right) \bar{\Phi}\left(\frac{x-\mu}{\sigma}\right)\left[1-\bar{\Phi}\left(\frac{x-\mu}{\sigma}\right)^{2}\right]^{\alpha \beta-1}}{\left\{1-\left[1-\bar{\Phi}\left(\frac{x-\mu}{\sigma}\right)^{2}\right]^{\alpha}\right\}^{\beta+1}} \exp \left\{-\left(\frac{\left[1-\bar{\Phi}\left(\frac{x-\mu}{\sigma}\right)^{2}\right]^{\alpha}}{1-\left[1-\bar{\Phi}\left(\frac{x-\mu}{\sigma}\right)^{2}\right]^{\alpha}}\right)^{\beta}, x \in R,\right.
$$

where $\phi(x)$ and $\Phi(x)$ are the pdf and cdf of the standard normal, respectively. If $X$ is a random variable with density function (2.1), then it will be denoted by WTLG$\mathrm{N}(\alpha, \beta, \mu, \sigma)$.

Figure 1 displays pdf's of WTLG-N for different parameter values. Figure 1 displays different skewed density functions including mild and high skewed ones (positive and negative). The WTLG-N family contains very flexible density function (unimodal and bimodal) that are useful in fitting real data sets. 

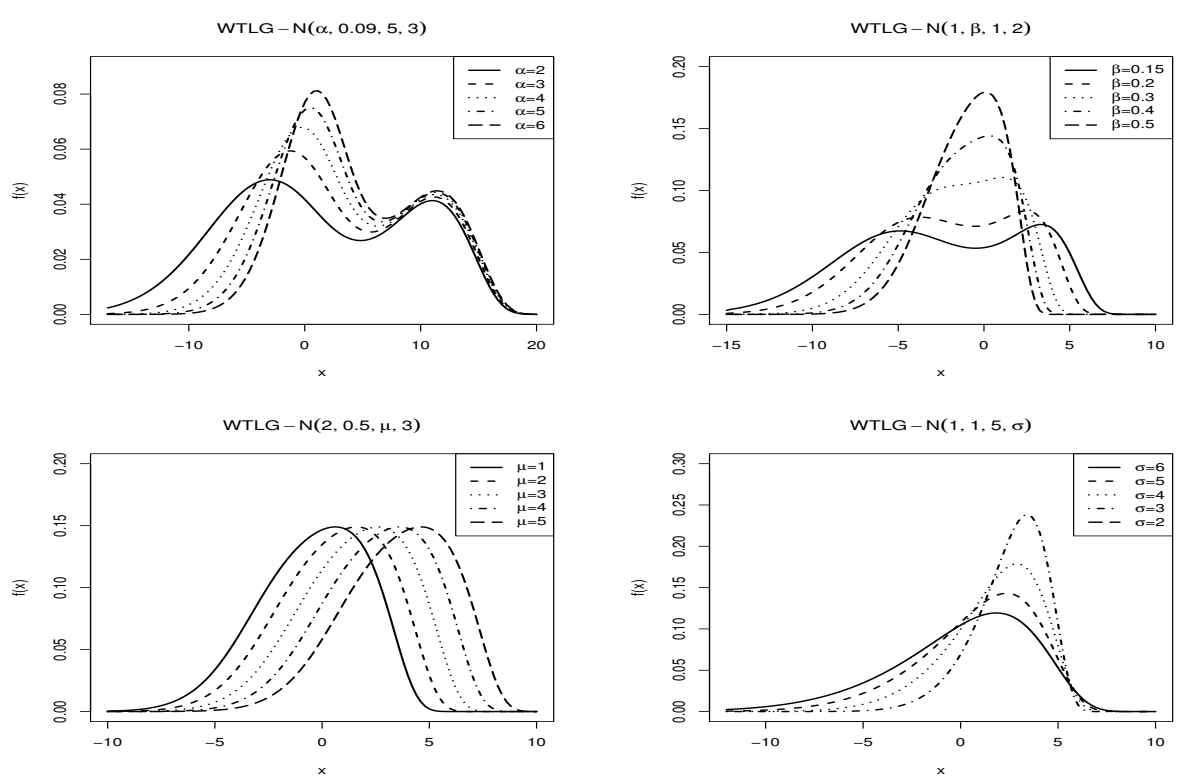

Figure 1: Plots of the density function of WTLG-N $(\alpha, \beta, \mu, \sigma)$ for selected parameter values.

\subsection{The Weibull Topp-Leone Generated Weibull Family (WTLG-W)}

Taking $G(x ; \psi)$ and $g(x ; \psi)$ as the cdf and pdf of the Weibull distribution with cdf $G(x ; \lambda, k)=1-e^{-\left(\frac{x}{\lambda}\right)^{b}}$ and pdf $g(x ; \lambda, b)=\frac{b}{\lambda}\left(\frac{x}{\lambda}\right)^{b-1} e^{-\left(\frac{x}{\lambda}\right)^{b}}$, in $((1.2))$, where $b>0$ is a shape parameter, $\lambda>0$ is a scale parameter, and $\xi=(\lambda, b)^{T}$, the WTLG-W density $(x>0)$ is

$$
f(x)=\frac{2 \alpha \beta \frac{b}{\lambda}\left(\frac{x}{\lambda}\right)^{b-1} e^{-2\left(\frac{x}{\lambda}\right)^{b}}\left[1-e^{-2\left(\frac{x}{\lambda}\right)^{k}}\right]^{\alpha \beta-1}}{\left\{1-\left[1-e^{-2\left(\frac{x}{\lambda}\right)^{b}}\right]^{\alpha}\right\}^{\beta+1}} \exp \left\{-\left(\frac{\left[1-e^{-2\left(\frac{x}{\lambda}\right)^{b}}\right]^{\alpha}}{1-\left[1-e^{-2\left(\frac{x}{\lambda}\right)^{b}}\right]^{\alpha}}\right)^{\beta}\right\} .
$$

Figure 2 displays density and hazed functions of WTLG-W for selected parameter values. 

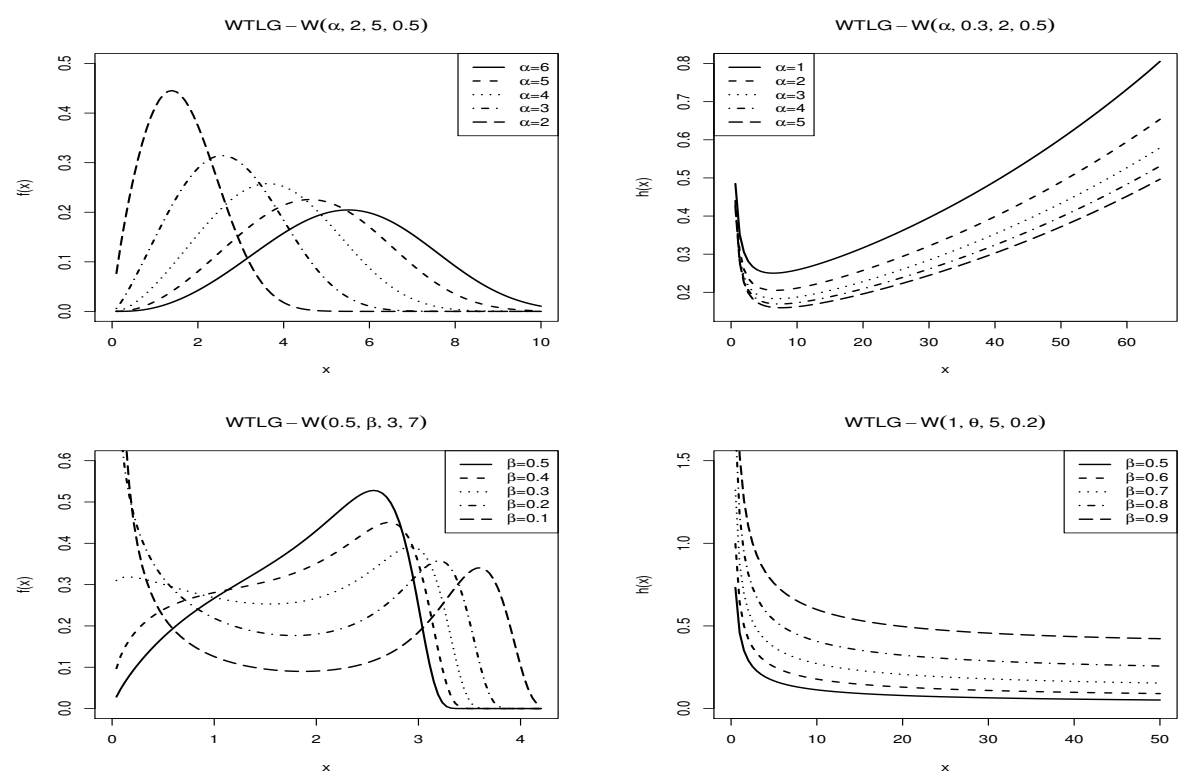

Figure 2: Plots of pdf and hazard functions of WTLG-W $(\alpha, \beta, \lambda, k)$ for selected parameter values.

\subsection{The Weibull Topp-Leone Generated Generalized Half Normal Family (WTLG-GHN)}

Taking $G(x ; \psi)$ and $g(x ; \psi)$ as the cdf and pdf of the generalized half normal distribution (GHN) (Cooray and Ananda (2008)) with cdf $G(x ; \theta, \lambda)=2 \Phi\left[\left(\frac{x}{\theta}\right)^{\lambda}\right]-1$ and pdf $g(x ; \theta, \lambda)=\sqrt{\frac{2}{\pi}}\left(\frac{\lambda}{x}\right)\left(\frac{x}{\theta}\right)^{\lambda} \exp \left[-\frac{1}{2}\left(\frac{x}{\theta}\right)^{2 \lambda}\right]$, in (1.2), with shape parameter $\lambda>0$ and scale parameter $\theta>0$, the WTLG-GHN density $(x>0)$ is

$$
f(x)=\frac{2 \alpha \beta \sqrt{\frac{2}{\pi}}\left(\frac{\lambda}{x}\right)\left(\frac{x}{\theta}\right)^{\lambda} \exp \left[-\frac{1}{2}\left(\frac{x}{\theta}\right)^{2 \lambda}\right]\left(2-2 \Phi\left[\left(\frac{x}{\theta}\right)^{\lambda}\right]\right)\left[1-\left(2-2 \Phi\left[\left(\frac{x}{\theta}\right)^{\lambda}\right]\right)^{2}\right]^{\alpha \beta-1}}{\left\{1-\left[1-\left(2-2 \Phi\left[\left(\frac{x}{\theta}\right)^{\lambda}\right]\right)^{2}\right]^{\alpha}\right\}^{\beta+1}}
$$




$$
\times \exp \left\{-\left(\frac{\left[1-\left(2-2 \Phi\left[\left(\frac{x}{\theta}\right)^{\lambda}\right]\right)^{2}\right]^{\alpha}}{1-\left[1-\left(2-2 \Phi\left[\left(\frac{x}{\theta}\right)^{\lambda}\right]\right)^{2}\right]^{\alpha}}\right)^{\beta}\right\}
$$

Figure 3 displays density and hazed functions of WTLG-GHN for selected parameter values.
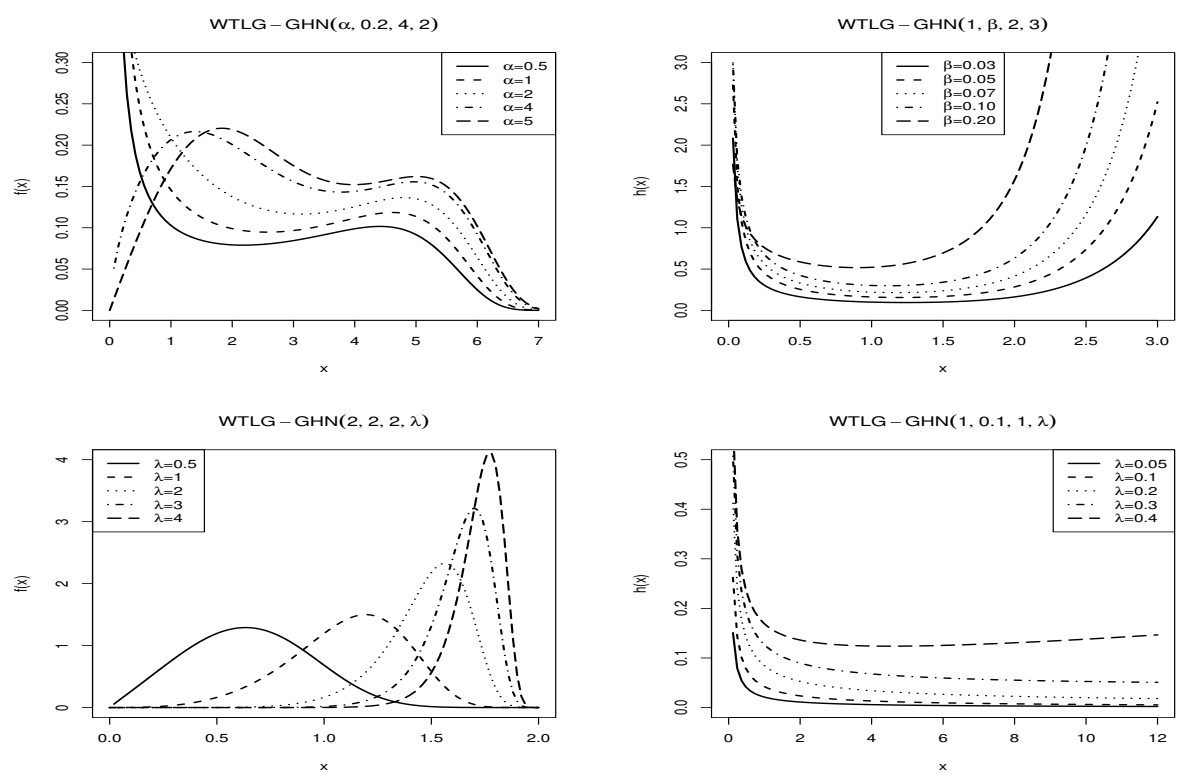

Figure 3: Plots of pdf and hazard functions of WTLG-GHN $(\alpha, \beta, \theta, \lambda)$ for selected parameter values.

\subsection{The Weibull Topp-Leone Generated Lindley Family (WTLG-L)}

Taking $G(x ; \psi)$ and $g(x ; \psi)$ as the cdf and pdf of the Lindley distribution (Lindley, 1958) with $\operatorname{cdf} G(x ; \theta)=1-\frac{\theta+1+\theta x}{\theta+1} e^{-\theta x}$ and pdf $g(x ; \theta)=\frac{\theta^{2}}{\theta+1}(x+1) e^{-\theta x}$, in ((1.2)), where $\theta>0$ 
and $x>0$, the WTLG-L density

$$
\begin{aligned}
f(x)= & \frac{2 \alpha \beta \frac{\theta^{2}}{\theta+1}(x+1) e^{-\theta x}\left(\frac{\theta+1+\theta x}{\theta+1} e^{-\theta x}\right)\left[1-\left(\frac{\theta+1+\theta x}{\theta+1} e^{-\theta x}\right)^{2}\right]^{\alpha \beta-1}}{\left\{1-\left[1-\left(\frac{\theta+1+\theta x}{\theta+1} e^{-\theta x}\right)^{2}\right]^{\alpha}\right\}^{\beta+1}} \\
& \times \exp \left\{-\left(\frac{\left[1-\left(\frac{\theta+1+\theta x}{\theta+1} e^{-\theta x}\right)^{2}\right]^{\alpha}}{1-\left[1-\left(\frac{\theta+1+\theta x}{\theta+1} e^{-\theta x}\right)^{2}\right]^{\alpha}}\right\}^{\beta}, \quad x \in \mathbb{R} .\right.
\end{aligned}
$$

Figure 4 displays density and hazed functions for WTLG-L for selected parameter values.
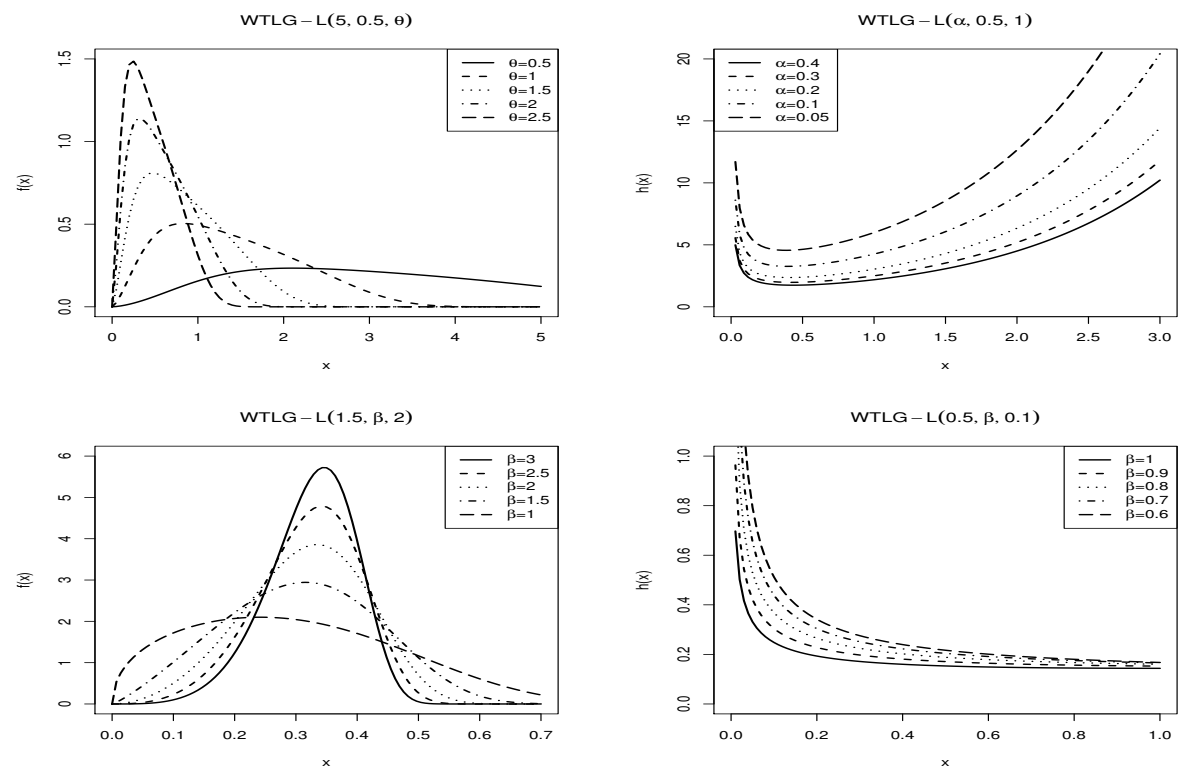

Figure 4: Plots of pdf and hazard functions of WTLG-L $(\alpha, \beta, \theta)$ for selected parameter values. 


\section{Some Mathematical Properties}

In this section, we first investigate asymptotic properties of this model and give mixture representations for cdf and pdf. Then, some mathematical properties of the new model such as limit behaviors, mixture representation, skewness and kurtosis, moments, moment generating function, incomplete moment are derived.

\subsection{Limit Behavior}

Proposition 3.1. Let $a=\inf \{x \mid F(x ; \psi)>0\}$, then, the asymptotic of equations (1.1), (1.2) and (1.3) as $x \rightarrow$ a are given by the following:

$$
\begin{aligned}
& F(x) \sim[2 G(x ; \psi)]^{\alpha \beta} \text { as } x \rightarrow a, \\
& f(x) \sim \alpha \beta 2^{\alpha \beta} g(x ; \psi) G(x ; \psi)^{\alpha \beta-1} \text { as } x \rightarrow a, \\
& h(x) \sim \alpha \beta 2^{\alpha \beta} g(x ; \psi) G(x ; \psi)^{\alpha \beta-1} \text { as } x \rightarrow a .
\end{aligned}
$$

Proposition 3.2. The asymptotic of equations (1.1), (1.2) and (1.3) as $x \rightarrow \infty$ are given by the following:

$$
\begin{aligned}
& \left.1-F(x) \sim \exp \left\{-\left[\alpha \bar{G}(x)^{2}\right]\right)^{-\beta}\right\} \text { as } x \rightarrow \infty, \\
& f(x) \sim 2 \beta \alpha^{-\beta} g(x ; \psi) \bar{G}(x ; \psi)^{-2 \beta-1} \exp \left\{-\left[\alpha \bar{G}(x)^{2}\right]^{-\beta}\right\} \text { as } x \rightarrow \infty, \\
& h(x) \sim 2 \beta \alpha^{-\beta} g(x ; \psi) \bar{G}(x ; \psi)^{-2 \beta-1} \text { as } x \rightarrow \infty .
\end{aligned}
$$

\subsection{Mixture Representation}

Using Taylor and generalized binomial expansions we have

$$
\begin{aligned}
F(x) & =\sum_{i=1}^{\infty}(-1)^{i+1}(i !)^{-1}\left\{\frac{\left[1-\bar{G}(x ; \psi)^{2}\right]^{\alpha}}{1-\left[1-\bar{G}(x ; \psi)^{2}\right]^{\alpha}}\right\}^{\beta i} \\
& =\sum_{i=1}^{\infty} \sum_{j=0}^{\infty}(-1)^{i+j+1}(i !)^{-1}\left(\begin{array}{c}
-\beta i \\
j
\end{array}\right)\left[1-\bar{G}(x ; \psi)^{2}\right]^{\alpha \beta i+\alpha j} \\
& =\sum_{i=1}^{\infty} \sum_{j, l=0}^{\infty}(-1)^{i+j+l+1}(i !)^{-1}\left(\begin{array}{c}
-\beta i \\
j
\end{array}\right)\left(\begin{array}{c}
(\beta i+j) \alpha \\
l
\end{array}\right) \bar{G}(x ; \psi)^{2 l}
\end{aligned}
$$




$$
\begin{aligned}
& =\sum_{i=1}^{\infty} \sum_{j, l=0}^{\infty} \sum_{d=0}^{2 l}(-1)^{i+j+l+d+1}(i !)^{-1}\left(\begin{array}{c}
-\beta i \\
j
\end{array}\right)\left(\begin{array}{c}
(\beta i+j) \alpha \\
l
\end{array}\right)\left(\begin{array}{c}
2 l \\
d
\end{array}\right) G(x ; \psi)^{d} \\
& =\sum_{d=0}^{2 l} w_{d} H_{d}(x ; \psi)
\end{aligned}
$$

where,

$$
w_{k}=\sum_{i=1}^{\infty} \sum_{j, l=0}^{\infty}(-1)^{i+j+l+d+1}(i !)^{-1}\left(\begin{array}{c}
-\beta i \\
j
\end{array}\right)\left(\begin{array}{c}
(\beta i+j) \alpha \\
l
\end{array}\right)\left(\begin{array}{c}
2 l \\
d
\end{array}\right),
$$

and $H_{d}(x ; \psi)=G(x ; \psi)^{d}$ is the cdf of the Exp-G family with power parameter $d$. Also, we can express the pdf $f(x)$ as follows:

$$
f(x)=\sum_{k=0}^{2 l} w_{d} h_{d}(x ; \psi)
$$

where $h_{d}(x)=d g(x ; \psi) G(x ; \psi)^{d-1}$ is the Exp-G pdf with power parameter $d$.

\subsection{Moments}

The $r$ th ordinary moment of $X$ is given by

$$
\mu_{r}^{\prime}=E\left(X^{r}\right)=\sum_{d=0}^{2 l} w_{d} E\left(Y_{d}^{r}\right)
$$

where $Y_{d}$ denotes the Exp-G distribution with power parameter $d$. Setting $r=1$ in (3.2), we have the mean of $X$. The last integration can be carried out numerically for most parent distributions. The skewness and kurtosis measures can be calculated from the ordinary moments using well-known relationships. The $n$th central moment of $X$, say $M_{n}$, is given by $M_{n}=E(X-\mu)^{n}=\sum_{h=0}^{n}(-1)^{h}\left(\begin{array}{l}n \\ h\end{array}\right)\left(\mu_{1}^{\prime}\right)^{n} \mu_{n-h}^{\prime}$. The cumulants $\left(\kappa_{n}\right)$ of $X$ follow recursively from $\kappa_{n}=\mu_{n}^{\prime}-\sum_{r=0}^{n-1}\left(\begin{array}{c}n-1 \\ r-1\end{array}\right) \kappa_{r} \mu_{n-r}^{\prime}$, where $\kappa_{1}=\mu_{1}^{\prime}, \kappa_{2}=\mu_{2}^{\prime}-\mu_{1}^{\prime 2}, \kappa_{3}=$ $\mu_{3}^{\prime}-3 \mu_{2}^{\prime} \mu_{1}^{\prime}+\mu_{1}^{\prime 3}$, and so on. For the WTLG-W model we have,

$$
\mu_{r}^{\prime}=\sum_{d=0}^{2 l} \sum_{h=0}^{\infty} \tau_{h, k}^{(r, d)} \Gamma\left(1+\frac{r}{k}\right), \forall r>-k \text {, where, } \tau_{h, d}^{(r, d)}=\tau_{h}^{(r, d)} w_{d} \text {, and }
$$




$$
\tau_{h}^{(r, d)}=d(-1)^{i} \lambda^{r}(h+1)^{-(r+k) / k}\left(\begin{array}{c}
d-1 \\
h
\end{array}\right)
$$

The measures of skewness and kurtosis of the WTLG-N distribution can be obtained as follows:

$$
\begin{gathered}
\operatorname{Skewness}(X)=\frac{\mu_{3}^{\prime}-3 \mu_{2}^{\prime} \mu_{1}^{\prime}+2\left(\mu_{1}^{\prime}\right)^{3}}{\left(\mu_{2}^{\prime}-\left(\mu_{1}^{\prime}\right)^{2}\right)^{\frac{3}{2}}}=\frac{\kappa_{3}}{\kappa_{2}^{(3 / 2)}}, \\
\operatorname{Kurtosis}(X)=\frac{\mu_{4}^{\prime}-4 \mu_{1}^{\prime} \mu_{3}^{\prime}+6\left(\mu_{1}^{\prime}\right)^{2} \mu_{3}^{\prime}-3\left(\mu_{1}^{\prime}\right)^{4}}{\kappa_{2}} .
\end{gathered}
$$

The skewness and kurtosis of WTLG-N are given in Table 1 for selected values of $\alpha, \beta$, $\mu$ and $\sigma$.

When the values of $\alpha, \beta$ and $\sigma$ are fixed, the skewness of WTLG-N increases as $\mu$ increases, and the distribution changes from left skewed to right skewed. When the values of $\alpha, \beta$ and $\mu$ are fixed, the skewness of WTLG-N decreases as $\sigma$ increases, and the distribution changes from right skewed to left skewed.

The value of kurtosis for different values of the parameter behaves differently. For example, when $(\alpha, \beta)=(2,2)$ and $\mu$ is fixed, the kurtosis of WTLG-N decreases as $\sigma$ increases. Plots of skewness and kurtosis of the WTLG-N distribution are displayed in Figure 5. vspace $2 \mathrm{~mm}$

\subsection{Moment Generating Function}

Here, we provide two formulae for the moment generating function $M_{X}(t)=E\left(\mathrm{e}^{t X}\right)$ of $X$. Clearly, the first one can be derived from equation (3.1) as $M_{X}(t)=\sum_{k=0}^{2 l} w_{k} M_{k}(t)$, where $M_{k}(t)$ is the mgf of $Y_{k}$. Hence, $M_{X}(t)$ can be determined from the Exp-G generating functions. 
Table 1: Skewness and kurtosis of WTLG-N for selected values of $\alpha, \beta, \mu$ and $\sigma$.

\begin{tabular}{|c|c|c|c|c|c|c|c|c|}
\hline \multirow[b]{2}{*}{$\alpha$} & \multirow[b]{2}{*}{$\beta$} & \multirow[b]{2}{*}{$\mu$} & \multicolumn{2}{|c|}{$\sigma=5$} & \multicolumn{2}{|c|}{$\sigma=7$} & \multicolumn{2}{|c|}{$\sigma=10$} \\
\hline & & & Skewness & Kurtosis & Skewness & Kurtosis & Skewness & Kurtosis \\
\hline \multirow[t]{9}{*}{0.5} & 0.5 & 5 & -0.324 & 2.257 & -0.310 & 2.444 & -0.128 & 1.639 \\
\hline & & 7 & 0.483 & 1.846 & 0.295 & 1.608 & 0.301 & 1.716 \\
\hline & & 10 & 0.817 & 2.708 & 0.723 & 2.425 & 0.558 & 2.146 \\
\hline & 1 & 5 & -0.235 & 2.156 & -0.211 & 2.280 & -0.103 & 1.627 \\
\hline & & 7 & 0.258 & 1.813 & 0.245 & 1.692 & 0.313 & 1.819 \\
\hline & & 10 & 0.429 & 2.037 & 0.326 & 1.997 & 0.302 & 1.968 \\
\hline & 2 & 5 & 0.033 & 1.688 & -0.158 & 1.806 & -0.291 & 1.792 \\
\hline & & 7 & 0.092 & 1.578 & $0 .-011$ & 1.648 & -0.019 & 1.799 \\
\hline & & 10 & 0.849 & 2.313 & 0.446 & 1.819 & 0.134 & 1.709 \\
\hline \multirow[t]{9}{*}{1} & 0.5 & 5 & -0.249 & 1.558 & -0.661 & 1.901 & -0.967 & 2.481 \\
\hline & & 7 & -0.140 & 1.808 & -0.501 & 2.265 & -0.800 & 2.840 \\
\hline & & 10 & 0.304 & 1.607 & -0.029 & 1.529 & -0.343 & 1.671 \\
\hline & 1 & 5 & 0.359 & 1.675 & -0.174 & 1.736 & -0.658 & 2.404 \\
\hline & & 7 & 0.425 & 1.686 & 0.0345 & 1.572 & -0.355 & 1.809 \\
\hline & & 10 & 1.075 & 2.742 & 0.653 & 1.993 & 0.276 & 1.656 \\
\hline & 2 & 5 & 0.867 & 2.223 & 0.406 & 1.663 & 0.007 & 1.554 \\
\hline & & 7 & 0.867 & 2.222 & 0.388 & 1.622 & 0.048 & 1.414 \\
\hline & & 10 & 2.025 & 6.003 & 1.519 & 4.071 & 1.041 & 2.775 \\
\hline \multirow[t]{9}{*}{2} & 0.5 & 5 & 0.662 & 1.945 & 0.252 & 1.448 & 0.182 & 1.333 \\
\hline & & 7 & 0.700 & 2.005 & 0.210 & 1.643 & -0.335 & 1.721 \\
\hline & & 10 & 0.720 & 1.963 & 0.251 & 1.565 & -0.143 & 1.663 \\
\hline & 1 & 5 & 1.323 & 3.296 & 0.843 & 2.227 & 0.326 & 1.672 \\
\hline & & 7 & 1.324 & 3.298 & 0.856 & 2.240 & 0.405 & 1.683 \\
\hline & & 10 & 1.792 & 4.687 & 1.282 & 3.050 & 0.768 & 1.967 \\
\hline & 2 & 5 & 1.888 & 5.209 & 1.378 & 3.449 & 0.878 & 2.277 \\
\hline & & 7 & 1.888 & 5.209 & 1.377 & 3.448 & 0.860 & 2.242 \\
\hline & & 10 & 3.575 & 15.621 & 2.899 & 10.818 & 2.273 & 7.266 \\
\hline
\end{tabular}



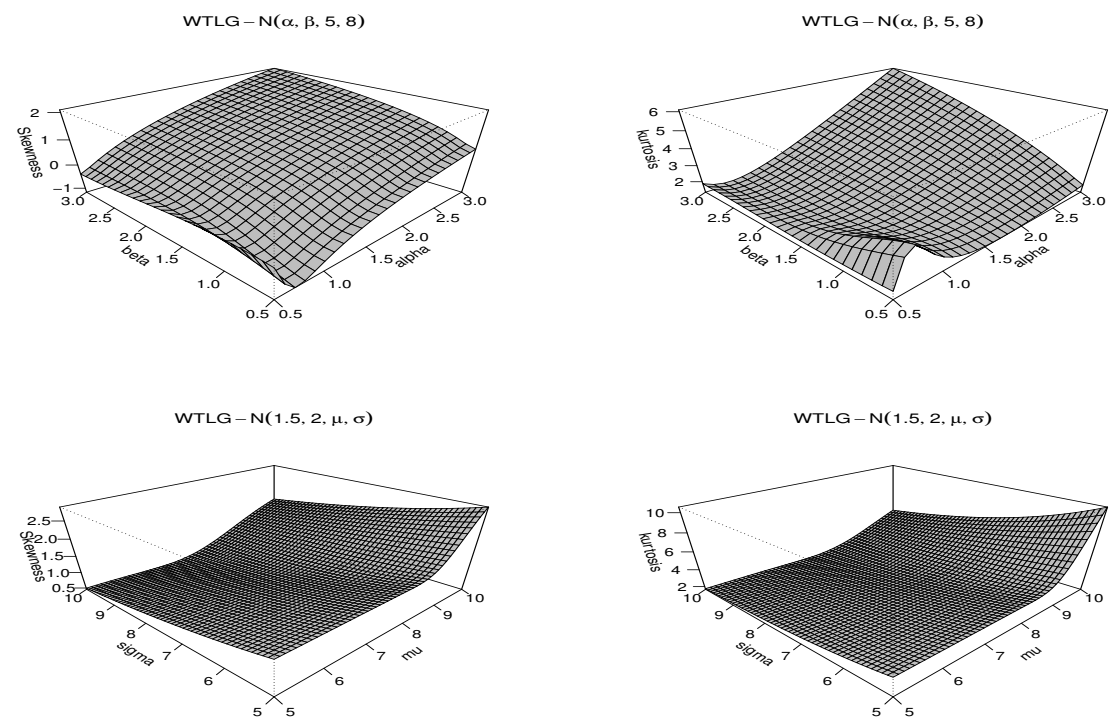

Figure 5: Skewness and Kurtosis for WTLG-N.

A second formula for $M_{X}(t)$ follows from (3.1) as $M_{X}(t)=\sum_{k=0}^{2 l} w_{k} \tau(t, k)$, where $\tau(t, k)=\int_{0}^{1} \exp \left[t Q_{G}(u)\right] u^{k} d u$ and $Q_{G}(u)$ is the quantile function (qf) corresponding to $G(x ; \psi)$, i.e., $Q_{G}(u)=G^{-1}(u ; \psi)$. The main application of the first incomplete moment refer to the mean deviations, and the Bonferroni and Lorenz curves. These curves are very useful in economics, reliability, demography, insurance, and medicine. For the WTLG-W model we have,

$$
M_{X}(t)=\sum_{d=0}^{2 l} \sum_{r, h=0}^{\infty} \Gamma(1+r / k), \forall r>-k \text {, where } \tau_{h, d, r}^{(r, d)}=\tau_{h}^{(r, d)}(r !)^{-1} t^{r} w_{d}
$$

\subsection{Incomplete Moment}

The sth incomplete moment, say $\varphi_{s}(t)$, of $X$ can be expressed from (3.1) as

$$
\varphi_{s}(t)=\int_{-\infty}^{t} x^{s} f(x) d x=\sum_{d=0}^{2 l} w_{d} \int_{-\infty}^{t} x^{s} h_{d}(x) d x
$$


The mean deviations about the mean and the median are as follows:

$$
\begin{gathered}
\delta_{1}=E\left(\left|X-\mu_{1}^{\prime}\right|\right)=2 \mu_{1}^{\prime} F\left(\mu_{1}^{\prime}\right)-2 \varphi_{1}\left(\mu_{1}^{\prime}\right), \\
\delta_{2}=E(|X-M|)=\mu_{1}^{\prime}-2 \varphi_{1}(M),
\end{gathered}
$$

where $\mu_{1}^{\prime}=E(X), M=\operatorname{Median}(X)=Q(0.5)$ is the median, $F\left(\mu_{1}^{\prime}\right)$ is easily calculated from (1.1) and $\varphi_{1}(t)$ is the first incomplete moment given by (3.5) with $s=1$. Now, we provide two ways to determine $\delta_{1}$ and $\delta_{2}$. First, a general equation for $\varphi_{1}(t)$ can be derived from (3.5) as $\varphi_{1}(t)=\sum_{d=0}^{2 l} w_{d} J_{d}(x)$, where $J_{d}(x)=\int_{-\infty}^{t} x h_{d}(x) d x$ is the first incomplete moment of the Exp-G distribution. A second general formula for $\varphi_{1}(t)$ is given by $\varphi_{1}(t)=\sum_{d=0}^{2 l} w_{d} v_{d}(t)$, where $v_{d}(t)=d \int_{0}^{G(t)} Q_{G}(u) u^{d-1} d u$ can be computed numerically. These equations for $\varphi_{1}(t)$ can be applied to construct Bonferroni and Lorenz curves defined for a given probability $\pi$ by $B(\pi)=\varphi_{1}(q)\left(\pi \mu_{1}^{\prime}\right)^{-1}$ and $L(\pi)=\varphi_{1}(q)\left(\mu_{1}^{\prime}\right)^{-1}$, respectively, where $\mu_{1}^{\prime}=E(X)$ and $q=Q(\pi)$ is the qf of $X$ at $\pi$. For the WTLG-W model we have,

$$
\begin{gathered}
\varphi_{s}(t)=\sum_{d=0}^{2 l} \sum_{h=0}^{\infty} \tau_{h, d}^{(s, d)} \gamma\left(1+s / k, \lambda t^{-k}\right), \forall s>-k, \\
\varphi_{1}(t)=\sum_{d=0}^{2 l} \sum_{h=0}^{\infty} \tau_{h, d}^{(1, d)} \gamma\left(1+1 / k, \lambda t^{-k}\right), \forall 1>-k,
\end{gathered}
$$

where,

$$
\begin{gathered}
\tau_{h, d}^{(s, k)}=\tau_{h}^{(s, d)} w_{d} \\
\tau_{h}^{(s, d)}=d(-1)^{i} \lambda^{s}(h+1)^{-(s+k) / k}\left(\begin{array}{c}
d-1 \\
h
\end{array}\right) \\
\gamma(p, z)=\int_{0}^{z} x^{p-1} e^{-x} d x
\end{gathered}
$$

\section{Characterizations Results}

In this section, we present certain characterizations of the WTLG distribution: (i) based on the ratio of two truncated moments; (ii) in terms of the hazard function and (iii) based on the conditional expectation of a certain function of the random variable. Note that $(i)$ can also be employed when the cdf does not have a closed form. We would 
also like to mention that due to the nature of WTLG distribution, our characterizations maybe the only possible ones. We present our characterizations (i) - (iii) in three subsections.

\subsection{Characterizations Based on Two Truncated Moments}

This subsection is devoted to the characterizations of WTLG distribution based on the ratio of two truncated moments. Our first characterization employs a theorem due to Glänzel (1987), see Theorem 1 of Appendix B. The result, however, holds also when the interval $H$ is not closed, since the condition of the Theorem is on the interior of $H$.

Proposition 4.1. Let $X: \Omega \rightarrow \mathbb{R}$ be a continuous random variable and let

$q_{1}(x)=\frac{\left\{1-\left[1-\bar{G}(x ; \psi)^{2}\right]^{\alpha}\right\}^{\beta+1}}{\left[1-\bar{G}(x ; \psi)^{2}\right]^{\alpha \beta-\alpha}} \exp \left\{\left[\frac{\left(1-\bar{G}(x ; \psi)^{2}\right)^{\alpha}}{1-\left(1-\bar{G}(x ; \psi)^{2}\right)^{\alpha}}\right]^{\beta}\right\}$ and $q_{2}(x)=q_{1}(x)\left[1-\bar{G}(x ; \psi)^{2}\right]^{\alpha}$ for $x \in$ $\mathbb{R}$. The random variable $X$ has $p d f(1.2)$ if and only if the function $\xi$ defined in Theorem 1 is of the form

$$
\xi(x)=\frac{1}{2}\left\{1+\left[1-\bar{G}(x ; \psi)^{2}\right]^{\alpha}\right\}, \quad x \in \mathbb{R} .
$$

Proof. Suppose the random variable $X$ has pdf (1.2), then

$$
(1-F(x)) E\left[q_{1}(X) \mid X \geq x\right]=\beta\left\{1-\left[1-\bar{G}(x ; \psi)^{2}\right]^{\alpha}\right\}, \quad x \in \mathbb{R},
$$

and

$$
(1-F(x)) E\left[q_{2}(X) \mid X \geq x\right]=\frac{\beta}{2}\left\{1-\left[1-\bar{G}(x ; \psi)^{2}\right]^{2 \alpha}\right\}, x \in \mathbb{R} .
$$

Further,

$$
\xi(x) q_{1}(x)-q_{2}(x)=\frac{1}{2}\left\{1-\left[1-\bar{G}(x ; \psi)^{2}\right]^{\alpha}\right\}>0, \text { for } x \in \mathbb{R} .
$$

Conversely, if $\xi$ is of the above form, then

$$
s^{\prime}(x)=\frac{\xi^{\prime}(x) q_{1}(x)}{\xi(x) q_{1}(x)-q_{2}(x)}=\frac{2 \alpha g(x ; \psi) \bar{G}(x ; \psi)\left[1-\bar{G}(x ; \psi)^{2}\right]^{\alpha-1}}{1-\left[1-\bar{G}(x ; \psi)^{2}\right]^{\alpha}},
$$

and consequently 


$$
s(x)=-\log \left(1-\left[1-\bar{G}(x ; \psi)^{2}\right]\right), \quad x \in \mathbb{R},
$$

Now, according to Theorem 1, X has pdf (1.2).

Corollary 4.1. Let $X: \Omega \rightarrow \mathbb{R}$ be a continuous random variable and let $q_{1}(x)$ be as in Proposition 4.1. The random variable $X$ has $p d f(1.2)$ if and only if there exist functions $q_{2}$ and $\xi$ defined in Theorem 1 satisfying the following differential equation

$$
\frac{\xi^{\prime}(x) q_{1}(x)}{\xi(x) q_{1}(x)-q_{2}(x)}=\frac{2 \alpha g(x ; \psi) \bar{G}(x ; \psi)\left[1-\bar{G}(x ; \psi)^{2}\right]^{\alpha-1}}{1-\left[1-\bar{G}(x ; \psi)^{2}\right]^{\alpha}}, x \in \mathbb{R} .
$$

Corollary 4.2. The general solution of the differential equation in Corollary 4.1 is

$$
\xi(x)=\left\{1-\left[1-\bar{G}(x ; \psi)^{2}\right]^{\alpha}\right\}^{-1}\left[\begin{array}{c}
-\int 2 \alpha g(x ; \psi) \bar{G}(x ; \psi)\left[1-\bar{G}(x ; \psi)^{2}\right]^{\alpha-1} \times \\
\left(q_{1}(x)\right)^{-1} q_{2}(x) d x+D
\end{array}\right],
$$

where $D$ is a constant. We like to point out that one set of functions satisfying the above differential equation is given in Proposition 4.1 with $D=\frac{1}{2}$. Clearly, there are other triplets $\left(q_{1}, q_{2}, \xi\right)$ which satisfy conditions of Theorem 1.

\subsection{Characterization in Terms of Hazard Function}

The hazard function, $h_{F}$, of a twice differentiable distribution function, $F$, satisfies the following first order differential equation

$$
\frac{f^{\prime}(x)}{f(x)}=\frac{h_{F}^{\prime}(x)}{h_{F}(x)}-h_{F}(x)
$$

It should be mentioned that for many univariate continuous distributions, the above equation is the only differential equation available in terms of the hazard function. In this subsection, we present a non-trivial characterization of WTLG distribution in terms of the hazard function.

Proposition 4.2. Let $X: \Omega \rightarrow \mathbb{R}$ be a continuous random variable. The random variable $X$ has $p d f(1.2)$ if and only if its hazard function $h_{F}(x)$ satisfies the following differential equation 


$$
h_{F}^{\prime}(x)+\frac{g(x ; \psi)}{\bar{G}(x ; \psi)} h_{F}(x)=2 \alpha \beta \bar{G}(x ; \psi) \frac{d}{d x}\left\{\frac{g(x ; \psi)\left[1-\bar{G}(x ; \psi)^{2}\right]^{\alpha \beta-1}}{\left\{1-\left[1-\bar{G}(x ; \psi)^{2}\right]^{\alpha}\right\}^{\beta+1}}\right\}, x \in \mathbb{R} .
$$

Proof. If $X$ has pdf (1.2), clearly then clearly the above differential equation holds. Now, if this equation holds, then

$$
\frac{d}{d x}\left\{\bar{G}(x ; \phi)^{-1} h_{F}(x)\right\}=2 \alpha \beta \frac{d}{d x}\left\{\frac{g(x ; \psi)\left[1-\bar{G}(x ; \psi)^{2}\right]^{\alpha \beta-1}}{\left\{1-\left[1-\bar{G}(x ; \psi)^{2}\right]^{\alpha}\right\}^{\beta+1}}\right\}, x \in \mathbb{R},
$$

from which we obtain the hazard function corresponding to the density (1.2).

\subsection{Characterization Based on the Conditional Expectation of a Certain Function of the Random Variable}

In this subsection, we employ a single function $\psi$ of $X$ and characterize the distribution of $X$ in terms of the truncated moment of $\psi(X)$. The following proposition has already appeared in Hamedani's previous work (2013), so we will just state it here which can be used to characterize WTLG distribution.

Proposition 4.3. Let $X: \Omega \rightarrow(e, f)$ be a continuous random variable with cdf $F$. Let $\psi(x)$ be a differentiable function on $(e, f)$ with $\lim _{x \rightarrow e^{+}} \psi(x)=1$. Then for $\delta \neq 1$,

$$
E[\psi(X) \mid X \geq x]=\delta \psi(x), \quad x \in(e, f)
$$

if and only if

$$
\psi(x)=(1-F(x))^{\frac{1}{\delta}-1}, \quad x \in(e, f)
$$

Remark 1. For $(e, f)=\mathbb{R}, \psi(x)=\exp \left\{-\left[\frac{\left(1-\bar{G}(x ; \psi)^{2}\right)^{\alpha}}{1-\left(1-\bar{G}(x ; \psi)^{2}\right)^{\alpha}}\right]^{\beta}\right\}$ and $\delta=\frac{1}{2}$, Proposition 4.3 provides a characterization of WTLG. 


\section{Maximum Llikelihood Estimators}

Several methods for parameter estimation exist but the maximum likelihood method is the most commonly employed. The MLE's enjoy desirable properties such as constructing confidence intervals for the model parameters. Large sample theory for these estimates deliver simple approximations that work well in finite samples. In this section the maximum likelihood equations for estimating the parameters are calculated. We evaluate the maximum likelihood estimation through the mean squared error and bias.

Let $x_{1}, \ldots, x_{n}$ be the observed values from the WTLG-W distribution with parameters $\alpha, \beta, \lambda$ and $k$. Let $\Theta=(\alpha, \beta, \lambda, k)^{\top}$ be the $4 \times 1$ parameter vector. The log-likelihood function for the vector of parameters $\Theta=(\alpha, \beta, \lambda, k)^{T}$, can be written as follows:

$$
\begin{aligned}
\ell(\Theta)= & n \ln 2+n \ln \alpha+n \ln \beta+\sum_{i=1}^{n} \ln g\left(x_{i} ; \psi\right)+\sum_{i=1}^{n} \ln \bar{G}\left(x_{i} ; \psi\right) \\
& +(\alpha \beta-1) \sum_{i=1}^{n} \ln \left[1-\bar{G}(x ; \psi)^{2}\right]-(\beta+1) \sum_{i=1}^{n} \ln \left\{1-\left[1-\bar{G}(x ; \psi)^{2}\right]^{\alpha}\right\} \\
& -\sum_{i=1}^{n}\left\{\frac{\left[1-\bar{G}(x ; \psi)^{2}\right]^{\alpha}}{1-\left[1-\bar{G}(x ; \psi)^{2}\right]^{\alpha}}\right\}^{\beta} .
\end{aligned}
$$

The log-likelihood function can be maximized either directly by solving the nonlinear likelihood equations obtained via differentiating (5.1).

The components of the score function $U_{n}(\Theta)=\left(\frac{\partial \ell(\Theta)}{\partial \alpha}, \frac{\partial \ell(\Theta)}{\partial \beta}, \frac{\partial \ell(\Theta)}{\partial \psi}\right)^{\top}$ are the following:

$$
\left\{\begin{aligned}
\frac{\partial \ln (\Theta)}{\partial \alpha}= & \frac{n}{\alpha}+\beta \sum_{i=1}^{n} \ln \left[1-\bar{G}\left(x_{i} ; \psi\right)^{2}\right]-\sum_{i=1}^{n} \frac{\beta \ln \left(1-\bar{G}\left(x_{i} ; \psi\right)^{2}\right)\left(\left(1-\bar{G}\left(x_{i} ; \psi\right)^{2}\right)^{\alpha}\right)^{\beta}}{\left\{1-\left[1-\bar{G}\left(x_{i} ; \psi\right)^{2}\right]^{\alpha}\right\}^{\beta+1}} \\
\frac{\partial \ln (\Theta)}{\partial \beta}= & \frac{n}{\beta}+\alpha \sum_{i=1}^{n} \ln \left[1-\bar{G}\left(x_{i} ; \psi\right)^{2}\right]-\sum_{i=1}^{n} \ln \left\{1-\left[1-\bar{G}\left(x_{i} ; \psi\right)^{2}\right]^{\alpha}\right\} \\
& -\sum_{i=1}^{n}\left(\frac{\left(1-\bar{G}\left(x_{i} ; \psi\right)^{2}\right)^{\alpha}}{1-\left(1-\bar{G}\left(x_{i} ; \psi\right)^{2}\right)^{\alpha}}\right)^{\beta} \ln \left(\frac{\left(1-\bar{G}\left(x_{i} ; \psi\right)^{2}\right)^{\alpha}}{1-\left(1-\bar{G}\left(x_{i} ; \psi\right)^{2}\right)^{\alpha}}\right) \\
\frac{\partial \ln (\Theta)}{\partial \psi}= & \sum_{i=1}^{n} \frac{g_{i}(\xi)}{g\left(x_{i} ; \psi\right)}-\sum_{i=1}^{n} \frac{G_{i}^{(\xi)}}{G\left(x_{i} ; \psi\right)}+2(\alpha \beta-1) \sum_{i=1}^{n} \frac{g\left(x_{i} ; \psi\right) \bar{G}\left(x_{i} ; \psi\right)}{1-\bar{G}\left(x_{i} ; \psi\right)^{2}} \\
& +(\beta+1) \sum_{i=1}^{n} \frac{2 \alpha g\left(x_{i} ; \psi\right)\left[1-\bar{G}\left(x_{i} ; \psi\right)^{2}\right]^{\alpha-1}}{1-\left[1-\bar{G}\left(x_{i} ; \psi\right)^{2}\right]^{\alpha}}-\sum_{i=1}^{n} \frac{2 \alpha \beta g\left(x_{i} ; \psi\right) \bar{G}\left(x_{i} ; \psi\right)\left[1-\bar{G}\left(x_{i} ; \psi\right)^{2}\right]^{\alpha \beta-1}}{\left\{1-\left[1-\bar{G}\left(x_{i} ; \psi\right)^{2}\right]^{\alpha}\right\}^{\beta+1}}
\end{aligned}\right.
$$


where $g_{i}^{(\xi)}=\frac{\partial g\left(x_{i} ; \psi\right)}{\partial \psi}$ and $G_{i}^{(\xi)}=\frac{\partial G\left(x_{i} ; \psi\right)}{\partial \psi}$.

\subsection{Maximum Likelihood Estimators of Censored Data}

Often with lifetime data, one encounters censored data. There are different forms of censoring: type $I$ censoring, type $I I$ censoring, etc. Here, we consider the general case of multicensored data: there are $n$ subjects of which

- $n_{0}$ are known to have failed at the times $x_{1}, x_{2}, \ldots, x_{n_{0}}$.

- $n_{1}$ are known to have failed in the interval $\left[s_{i-1}, s_{i}\right], i=1, \ldots, n_{1}$.

- $n_{2}$ survived to a time $r_{i}, i=1, \ldots, n_{2}$ but not observed any longer.

Note that $n=n_{0}+n_{1}+n_{2}$. Note also that type $I$ censoring and type $I I$ censoring are contained as particular cases of multi censoring. The log-likelihood function for the vector of parameters $\Theta=(\alpha, \beta, \lambda, k)^{T}$ for this multi censoring data is as follows;

$$
\begin{aligned}
\ell(\Theta)= & n_{0} \ln 2+n_{0} \ln \alpha+n_{0} \ln \beta+\sum_{i=1}^{n_{0}} \ln g\left(x_{i} ; \psi\right)+\sum_{i=1}^{n_{0}} \ln \bar{G}\left(x_{i} ; \psi\right) \\
& +(\alpha \beta-1) \sum_{i=1}^{n_{0}} \ln \left[1-\bar{G}(x ; \psi)^{2}\right]-(\beta+1) \sum_{i=1}^{n_{0}} \ln \left\{1-\left[1-\bar{G}(x ; \psi)^{2}\right]^{\alpha}\right\} \\
& -\sum_{i=1}^{n_{0}}\left\{\frac{\left[1-\bar{G}(x ; \psi)^{2}\right]^{\alpha}}{1-\left[1-\bar{G}(x ; \psi)^{2}\right]^{\alpha}}\right\}^{\beta} \\
& +\sum_{i=1}^{n_{1}}\left\{\left\{\frac{\left[1-\bar{G}\left(s_{i} ; \psi\right)^{2}\right]^{\alpha}}{1-\left[1-\bar{G}\left(s_{i} ; \psi\right)^{2}\right]^{\alpha}}\right\}^{\beta}-\left\{\frac{\left[1-\bar{G}\left(s_{i-1} ; \psi\right)^{2}\right]^{\alpha}}{\left.1-\left[1-\bar{G}\left(s_{i-1} ; \psi\right)^{2}\right]^{\alpha}\right\}}\right.\right. \\
& -\sum_{i=1}^{n_{2}}\left\{\frac{\left[1-\bar{G}\left(r_{i} ; \psi\right)^{2}\right]^{\alpha}}{1-\left[1-\bar{G}\left(r_{i} ; \psi\right)^{2}\right]^{\alpha}}\right\} .
\end{aligned}
$$


It follows that the MLEs are the simultaneous solutions of the equations:

$$
\begin{aligned}
& \int \frac{\partial \ln (\Theta)}{\partial \alpha}=\frac{n_{0}}{\alpha}+\beta \sum_{i=1}^{n_{0}} \ln \left[1-\bar{G}\left(x_{i} ; \psi\right)^{2}\right]-\sum_{i=1}^{n_{0}} \frac{\beta \ln \left(1-\bar{G}\left(x_{i} ; \psi\right)^{2}\right)\left(\left(1-\bar{G}\left(x_{i} ; \psi\right)^{2}\right)^{\alpha}\right)^{\beta}}{\left\{1-\left[1-\bar{G}\left(x_{i} ; \psi\right)^{2}\right]^{\alpha}\right\}^{\beta+1}} \\
& +\sum_{i=1}^{n_{1}}\left\{\frac{\beta \ln \left(1-\bar{G}\left(s_{i} ; \psi\right)^{2}\right)\left(\left(1-\bar{G}\left(s_{i} ; \psi\right)^{2}\right)^{\alpha}\right)^{\beta}}{\left\{1-\left[1-\bar{G}\left(s_{i} ; \psi\right)^{2}\right]^{\alpha}\right\}^{\beta+1}}\right. \\
& \left.-\frac{\beta \ln \left(1-\bar{G}\left(s_{i-1} ; \psi\right)^{2}\right)\left(\left(1-\bar{G}\left(s_{i-1} ; \psi\right)^{2}\right)^{\alpha}\right)^{\beta}}{\left\{1-\left[1-\bar{G}\left(s_{i-1} ; \psi\right)^{2}\right]^{\alpha}\right\}^{\beta+1}}\right\} \\
& -\sum_{i=1}^{n_{2}} \frac{\beta \ln \left(1-\bar{G}\left(r_{i} ; \psi\right)^{2}\right)\left(\left(1-\bar{G}\left(r_{i} ; \psi\right)^{2}\right)^{\alpha}\right)^{\beta}}{\left\{1-\left[1-\bar{G}\left(r_{i} ; \psi\right)^{2}\right]^{\alpha}\right\}^{\beta+1}} \text {. } \\
& \frac{\partial \ln (\Theta)}{\partial \beta}=\frac{n_{0}}{\beta}+\alpha \sum_{i=1}^{n_{0}} \ln \left[1-\bar{G}\left(x_{i} ; \psi\right)^{2}\right]-\sum_{i=1}^{n_{0}} \ln \left\{1-\left[1-\bar{G}\left(x_{i} ; \psi\right)^{2}\right]^{\alpha}\right\} \\
& -\sum_{i=1}^{n_{0}}\left(\frac{\left(1-\bar{G}\left(x_{i} ; \psi\right)^{2}\right)^{\alpha}}{1-\left(1-\bar{G}\left(x_{i} ; \psi\right)^{2}\right)^{\alpha}}\right)^{\beta} \ln \left(\frac{\left(1-\bar{G}\left(x_{i} ; \psi\right)^{2}\right)^{\alpha}}{1-\left(1-\bar{G}\left(x_{i} ; \psi\right)^{2}\right)^{\alpha}}\right) \\
& +\sum_{i=1}^{n_{1}}\left\{\left(\frac{\left(1-\bar{G}\left(s_{i} ; \psi\right)^{2}\right)^{\alpha}}{1-\left(1-\bar{G}\left(s_{i} ; \psi\right)^{2}\right)^{\alpha}}\right)^{\beta} \ln \left(\frac{\left(1-\bar{G}\left(s_{i} ; \psi\right)^{2}\right)^{\alpha}}{1-\left(1-\bar{G}\left(s_{i} ; \psi\right)^{2}\right)^{\alpha}}\right)\right. \\
& \left.-\left(\frac{\left(1-\bar{G}\left(s_{i-1} ; \psi\right)^{2}\right)^{\alpha}}{1-\left(1-\bar{G}\left(s_{i-1} ; \psi\right)^{2}\right)^{\alpha}}\right)^{\beta} \ln \left(\frac{\left(1-\bar{G}\left(s_{i-1} ; \psi\right)^{2}\right)^{\alpha}}{1-\left(1-\bar{G}\left(s_{i-1} ; \psi\right)^{2}\right)^{\alpha}}\right)\right\} \\
& -\sum_{i=1}^{n_{2}}\left(\frac{\left(1-\bar{G}\left(r_{i} ; \psi\right)^{2}\right)^{\alpha}}{1-\left(1-\bar{G}\left(r_{i} ; \psi\right)^{2}\right)^{\alpha}}\right)^{\beta} \ln \left(\frac{\left(1-\bar{G}\left(r_{i} ; \psi\right)^{2}\right)^{\alpha}}{1-\left(1-\bar{G}\left(r_{i} ; \psi\right)^{2}\right)^{\alpha}}\right) . \\
& \frac{\partial \ln (\Theta)}{\partial \psi}=\sum_{i=1}^{n_{0}} \frac{g_{i}{ }^{(\xi)}}{g\left(x_{i} ; \psi\right)}-\sum_{i=1}^{n_{0}} \frac{G_{i}{ }^{(\xi)}}{G\left(x_{i} ; \psi\right)}+2(\alpha \beta-1) \sum_{i=1}^{n_{0}} \frac{g\left(x_{i} ; \psi\right) \bar{G}\left(x_{i} ; \psi\right)}{1-\bar{G}\left(x_{i} ; \psi\right)^{2}} \\
& +(\beta+1) \sum_{i=1}^{n_{0}} \frac{2 \alpha g\left(x_{i} ; \psi\right)\left[1-\bar{G}\left(x_{i} ; \psi\right)^{2}\right]^{\alpha-1}}{1-\left[1-\bar{G}\left(x_{i} ; \psi\right)^{2}\right]^{\alpha}}-\sum_{i=1}^{n_{0}} \frac{2 \alpha \beta g\left(x_{i} ; \psi\right) \bar{G}\left(x_{i} ; \psi\right)\left[1-\bar{G}\left(x_{i} ; \psi\right)^{2}\right]^{\alpha \beta-1}}{\left\{1-\left[1-\bar{G}\left(x_{i} ; \psi\right)^{2}\right]^{\alpha}\right\}^{\beta+1}} \\
& +\sum_{i=1}^{n_{1}}\left\{\frac{2 \alpha \beta g\left(s_{i} ; \psi\right) \bar{G}\left(s_{i} ; \psi\right)\left[1-\bar{G}\left(s_{i} ; \psi\right)^{2}\right]^{\alpha \beta-1}}{\left\{1-\left[1-\bar{G}\left(s_{i} ; \psi\right)^{2}\right]^{\alpha}\right\}^{\beta+1}}\right. \\
& \left.-\frac{2 \alpha \beta g\left(s_{i-1} ; \psi\right) \bar{G}\left(s_{i-1} ; \psi\right)\left[1-\bar{G}\left(s_{i-1} ; \psi\right)^{2}\right]^{\alpha \beta-1}}{\left\{1-\left[1-\bar{G}\left(s_{i-1} ; \psi\right)^{2}\right]^{\alpha}\right\}^{\beta+1}}\right\} \\
& -\sum_{i=1}^{n_{2}} \frac{2 \alpha \beta g\left(r_{i} ; \psi\right) \bar{G}\left(r_{i} ; \psi\right)\left[1-\bar{G}\left(r_{i} ; \psi\right)^{2}\right]^{\alpha \beta-1}}{\left\{1-\left[1-\bar{G}\left(r_{i} ; \psi\right)^{2}\right]^{\alpha}\right\}^{\beta+1}} .
\end{aligned}
$$


where $g_{i}^{(\xi)}=\frac{\partial g\left(x_{i} ; \psi\right)}{\partial \psi}$ and $G_{i}^{(\xi)}=\frac{\partial G\left(x_{i} ; \psi\right)}{\partial \psi}$.

\section{Likelihood Ratio Test}

In this section, we apply likelihood ratio test (LRT) for testing the Weibull ToppLeone Generated Weibull distribution (WTLG-W) against the other known lifetime distributions such as The Zografos-Balakrishnan Weibull distribution (ZBW), MarshallOlkin Weibull distribution (MOW), and Weibull distribution.

The likelihood ratio statistic can be defined as follows:

$$
\Lambda(\underset{\sim}{x})=\frac{\sup _{\theta \in \Theta_{0}} L(\underset{\sim}{x}, \theta)}{\sup _{\theta \in \Theta_{1}} L(\underset{\sim}{x}, \theta)}=\frac{L(\underset{\sim}{x}, \hat{\theta})}{L(\underset{\sim}{x}, \hat{\hat{\theta}})},
$$

where $L \underset{\sim}{x}, \theta)$ is the likelihood function, $\hat{\theta}, \hat{\hat{\theta}}$ are maximum likelihood estimates of $\theta$ under $H_{0}$ and $H_{1}$ (null and alternative hypothesis) and $x$ is the vector of the observations.

We calculate the statistic $\lambda=-2 \ln \Lambda$, which has an approximate chi-square distribution with $r$ degrees of freedom (df), where $r=$ (df of alternative hypothesis) - (df of null hypothesis) by the LRT, we reject the null hypothesis, if $\lambda$ is larger than a chi-square 100(1- $\alpha)$ percentile with $r$ degree of freedom $\left(\chi_{1-\alpha}^{2}(r)\right)$. We use LRT to check if the fit using WTLG-W distribution is statistically superior to the fits using the ZBW, MOW and Weibull distributions. In fact, the test is as follows:

$$
\begin{cases}H_{0}: & \underset{\sim}{x} \sim \text { ZBW or MOWor Weibull, } \\ H_{1}: & \underset{\sim}{x} \sim \text { WTLG }-W .\end{cases}
$$

\section{Simulation Study}

\subsection{The Maximum Likelihood Estimator}

In this section, the maximum likelihood estimators of the parameters of the proposed density function have been assessed by simulating: $(\alpha, \beta, \lambda, k)=(2,2,3,2)$. The density function has been displayed in Figure 6 .

To verify the validity of the maximum likelihood estimator, the bias and the mean square error of MLE have been used. For example, for $(\alpha, \beta, \lambda, k)=(2,2,3,2), r=1000$ 
times have been simulated samples of $n=20,21, \ldots, 110$ of WTLG-W(2,2,3,2). To estimate the numerical value of the maximum likelihood, the optim function (in the stat package) and Nelder-Mead method in R software have been used. If $\theta=(\alpha, \beta, \lambda, k)$, for any simulation by $n$ volume and $i=1,2, \ldots, r$, the maximum likelihood estimates are obtained as $\widehat{\boldsymbol{\theta}}_{i}=\left(\widehat{\alpha}_{i}, \widehat{\beta}_{i}, \widehat{\lambda}_{i}, \widehat{k}_{i}\right)$.

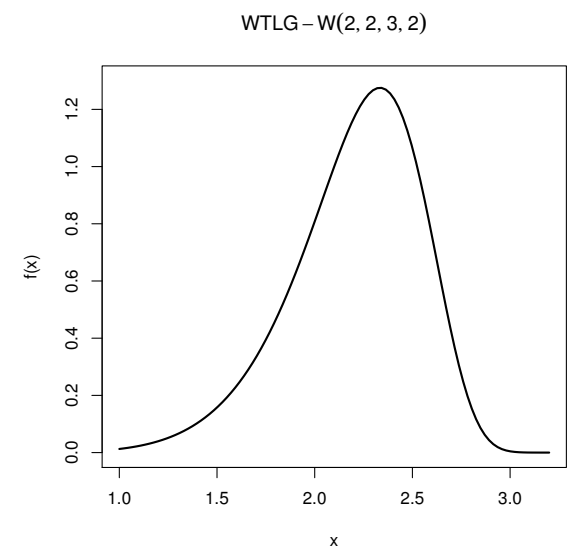

Figure 6: The density function for the study.

To examine the performance of the MLE's for the WTLG-W distribution, we perform a simulation study:

1. Generate $r$ samples of size $n$ from equation (1.2).

2. Compute the MLE's for the $r$ samples, say $(\hat{\alpha}, \hat{\beta}, \hat{\lambda}, \hat{k})$ for $i=1,2, \ldots, r$.

3. Compute the standard errors of the MLE's for $r$ samples, say $\left(s_{\hat{\alpha}}, s_{\hat{\beta}}, s_{\hat{\lambda}}, s_{\hat{k}}\right)$ for $i=1,2, \ldots, r$.

4. Compute the biases and mean squared errors given by $\left.\operatorname{Bias}_{\widehat{\boldsymbol{\theta}}}(n)=\frac{1}{r} \sum_{i=1}^{r} \widehat{\boldsymbol{\theta}}_{i}-\boldsymbol{\theta}_{i}\right)$ and $M S E_{\widehat{\boldsymbol{\theta}}}(n)=\frac{1}{r} \sum_{i=1}^{r}\left(\widehat{\boldsymbol{\theta}}_{i}-\boldsymbol{\theta}_{i}\right)^{2}$,for $\theta=(\alpha, \beta, \lambda, k)$. We repeat these steps for $r=1000$ and $n=20,21, \ldots n^{*}\left(n^{*}\right.$ is different in each issue) with different values of $(\alpha, \beta, \lambda, k)$, so computing $\operatorname{Bias}_{\widehat{\boldsymbol{\theta}}}(n), M S E_{\widehat{\boldsymbol{\theta}}}(n)$. Figure 5, 6 respectively reveals how the four 
biases, mean squared errors vary with respect to $n$. As expected, the Biases and MSE's of estimated parameters converge to zero as $n$ increases.
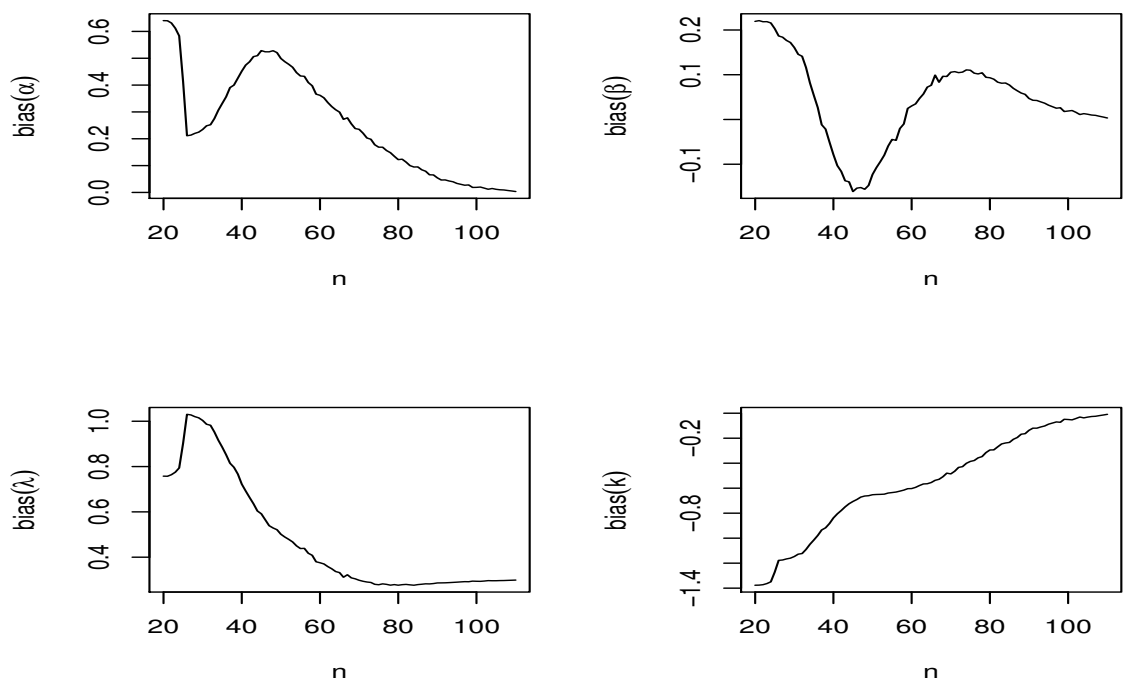

Figure 7: Bias of $\hat{\alpha}, \hat{\beta}, \hat{\lambda}, \hat{k}$ versus $n$ when $(\alpha, \beta, \lambda, k)=(2,2,3,2)$.

\subsection{The Other Estimation Methods}

There are several approaches to estimating the parameters of distributions, each of which has its own characteristic features and benefits. In this subsection five of those methods are briefly introduced and will be numerically investigated in the simulation study Figure 4. A useful summary of these methods can be seen in Dey et al. (2017). Here, $\left\{t_{i} ; i=1,2, \ldots, n\right\}$ is the associated order statistics and $F$ is the distribution function of WTLG-W.

\subsubsection{Least Squares and Weighted Least Squares Estimators}

The Least Squares (LSE) and Weighted Least Squares Estimators (WLSE) were introduced by Swain et al. (1988). The LSE's and WLSE's are obtained by minimizing the 
following functions:

$$
S_{\mathrm{LSE}}(\alpha, \beta, \lambda, k)=\sum_{i=1}^{n}\left[F\left(t_{i} ; \alpha, \beta, \lambda, k\right)-i(n+1)^{-1}\right]^{2},
$$

$S_{\mathrm{WLSE}}(\alpha, \beta, \lambda, k)=\sum_{i=1}^{n} \frac{(n+1)^{2}(n+2)}{i(n-i+1)}\left[F\left(t_{i} ; \alpha, \beta, \lambda, k\right)-i(n+1)^{-1}\right]^{2}$.
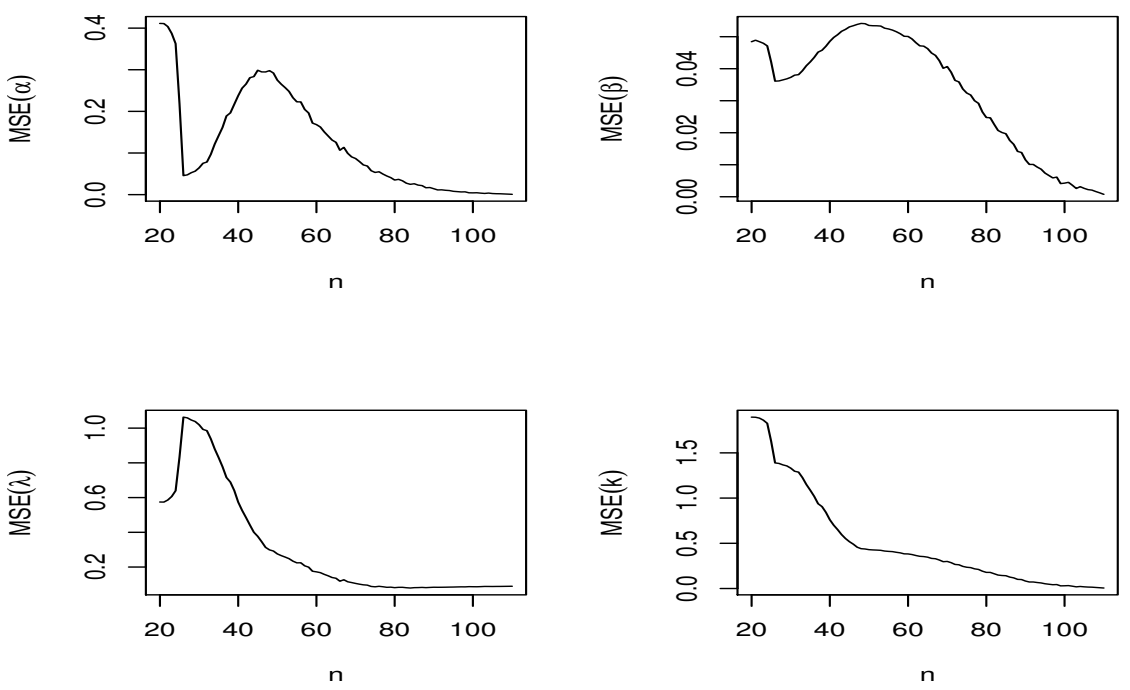

Figure 8: MSE of $\hat{\alpha}, \hat{\beta}, \hat{\lambda}, \hat{k}$ versus $n$ when $(\alpha, \beta, \lambda, k)=(2,2,3,2)$.

\subsubsection{Cramér-von-Mises Estimator}

Cramer-von Mise's Estimator (CME) was introduced by Choi and Bulgren (1968). The CME's is obtained by minimizing the following function:

$$
S_{\mathrm{CME}}(\alpha, \beta, \lambda, k)=\frac{1}{12 n}+\sum_{i=1}^{n}\left[F\left(t_{i} ; \alpha, \beta, \lambda, k\right)-(2 i-1)(2 n)^{-1}\right]^{2} \text {. }
$$




\subsubsection{Anderson-Darling and Right-tailed Anderson-Darling}

The Anderson-Darling (ADE) and Right-Tailed Anderson-Darling Estimators (RTADE) were introduced by Anderson and Darling (1952). The ADE's and RTADE's are obtained by minimizing the following functions:

$$
\begin{aligned}
& S_{\mathrm{ADE}}(\alpha, \beta, \lambda, k)=-n-\frac{1}{n} \sum_{i=1}^{n}(2 i-1)\left\{\log F\left(t_{i} ; \alpha, \beta, \lambda, k\right)+\log \bar{F}\left(t_{n+1-i} ; \alpha, \beta, \lambda, k\right)\right\}, \\
& S_{\mathrm{RTADE}}(\alpha, \beta, \lambda, k)=\frac{n}{2}-2 \sum_{i=1}^{n} F\left(t_{i} ; \alpha, \beta, \lambda, k\right)-\frac{1}{n} \sum_{i=1}^{n}(2 i-1) \log \bar{F}\left(t_{n+1-i} ; \alpha, \beta, \lambda, k\right) .
\end{aligned}
$$

In order to explore the estimators introduced above, we consider the model that has been used in this section and investigate bias and MSE of those estimators for different samples. For instance, according to what has been mentioned above, for $(\alpha, \beta, \lambda, k)=(2,2,3,2)$.

We have simulated $r=1000$ times with sample size of the $n=50,55,60, \cdots, 600$ and then the bias and MSE formula, mentioned in Section 7, are calculated for them. To obtain the values of the estimators, we have used the optima function and NelderMead method in R. The result of the simulations of this subsection is shown in Figures 9 and 10. It is clear from the bias and MSE plot for two parameters with the increase in the volume of the sample via all methods will approach zero and this verifies the validity of these estimation methods and numerical calculations for the parameters of WTLG-W.

\section{Applications}

In this section, we present two applications by fitting the WTLG-W as well as some well-known models. The Akaike information criterion (AIC), Bayesian information criterion (BIC), Cramer-von Mises $\left(W^{*}\right)$, Anderson-Darling $\left(A^{*}\right)$, Kolmogorov Smirnov (K.S) and the p-Value of K.S test, have been chosen for comparison of models for the two examples.

In general, the smaller the values of these statistics, the better fit to the data. 

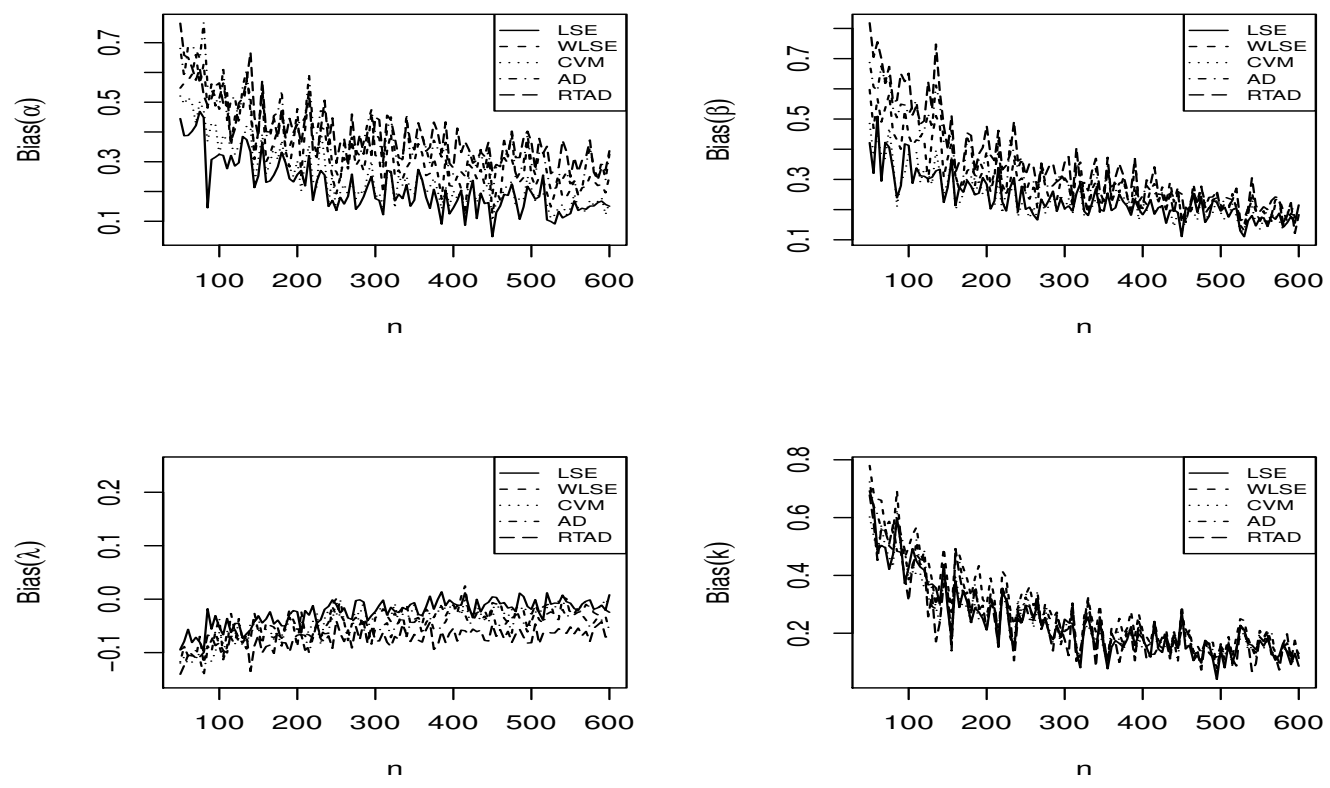

Figure 9: Bias of $\hat{\alpha}, \hat{\beta}, \hat{\lambda}, \hat{k}$ versus $n$ when $(\alpha, \beta, \lambda, k)=(2,2,3,2)$.
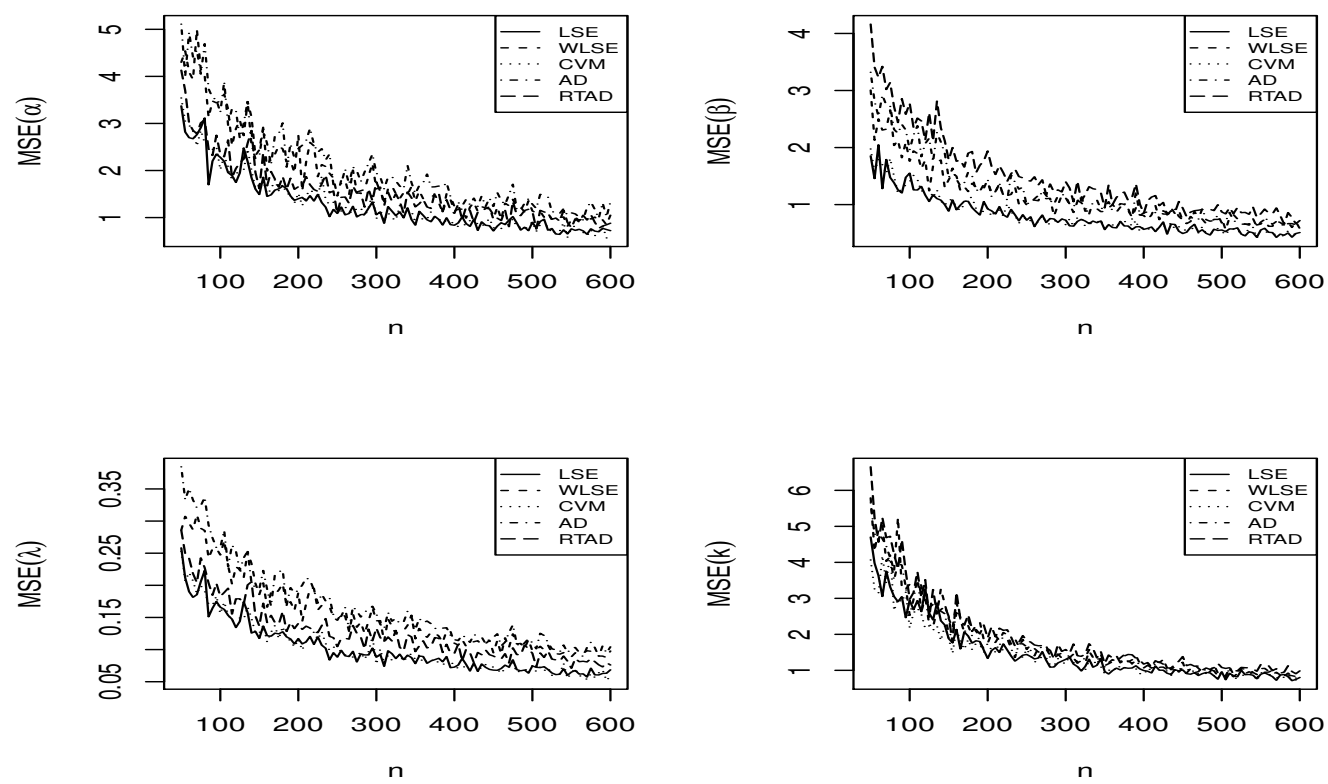

Figure 10: MSE of $\hat{\alpha}, \hat{\beta}, \hat{\lambda}, \hat{k}$ versus $n$ when $(\alpha, \beta, \lambda, k)=(2,2,3,2)$. 
The Gamma-Weibull distribution (GaW), Alzaatreh et al. (2014), The Beta-Weibull distribution (BW), Famoye et al. (2005), The McDonald Weibull distribution (McW), Hashimoto et al. (2015), Marshall-Olkin Weibull distribution (MOW), Pogany et al. (2015), The Kumaraswamy Weibull distribution (KwW), Cordeiro et al. (2010) and The Zografos-Balakrishnan Weibull distribution (ZBW) Nadarajah et al. (2014), have been selected for comparison via two examples. The parameters of the models have been estimated by the MLE method.

\subsection{Phosphorus Concentration in Leaves Data Set.}

This sub-section is related to the study of the soil fertility in influence and the characterization of the biologic fixation of $N_{2}$ for the Dimorphandra wilsonii rizz growth. For 128 plants, they made measures of the phosphorus concentration in the leaves (Fonseca and Franca (2007)). The data, which have also been analyzed by Silva et al. (2013). The data set is the following: $0.22,0.17,0.11,0.10,0.15,0.06,0.05,0.07,0.12,0.09,0.23$, $0.25,0.23,0.24,0.20,0.08,0.11,0.12,0.10,0.06,0.20,0.17,0.20,0.11,0.16,0.09,0.10,0.12$, $0.12,0.10,0.09,0.17,0.19,0.21,0.18,0.26,0.19,0.17,0.18,0.20,0.24,0.19,0.21,0.22,0.17$, $0.08,0.08,0.06,0.09,0.22,0.23,0.22,0.19,0.27,0.16,0.28,0.11,0.10,0.20,0.12,0.15,0.08$, $0.12,0.09,0.14,0.07,0.09,0.05,0.06,0.11,0.16,0.20,0.25,0.16,0.13,0.11,0.11,0.11,0.08$, $0.22,0.11,0.13,0.12,0.15,0.12,0.11,0.11,0.15,0.10,0.15,0.17,0.14,0.12,0.18,0.14,0.18$, $0.13,0.12,0.14,0.09,0.10,0.13,0.09,0.11,0.11,0.14,0.07,0.07,0.19,0.17,0.18,0.16,0.19$, $0.15,0.07,0.09,0.17,0.10,0.08,0.15,0.21,0.16,0.08,0.10,0.06,0.08,0.12,0.13$.

We apply the WTLG-W to fit the data set and the results are given in Table 2 and Table 3. In Tables 2 and 3, a summary of the fitted information criteria and estimated MLE's for this data with different models are shown, respectively.

The AIC, BIC, $W^{*}, A^{*}, \mathrm{~K} . \mathrm{S}$ and P-Value (in Table 2) for the eight distributions are taken. Models have been sorted from the lowest to the highest value of AIC. As you see, the WTLG-W is selected as the best model with all criteria. The histogram of the Phosphorus concentration in leaves data and the plots of fitted pdf for the best model are displayed in Figure 11. In Figure 12, the plot of uni-modality of profile likelihood functions of parameters of WTLG-W $(\alpha, \beta, \lambda, k)$ is shown.

In Table 4 , information about the hypothesis test is at $0.05 \%$ for the test hypothesis in equation (6.1). As indicated in Table 4, the null hypothesis is rejected. 
Table 2: Information criteria for the Phosphorus concentration in leaves data.

\begin{tabular}{l|cccccc}
\hline Model & AIC & BIC & $W^{*}$ & $A^{*}$ & K.S & P-Value \\
\hline WTLG-W & -394.47 & -383.06 & 0.05 & 0.30 & 0.06 & 0.797 \\
ZBW & -387.81 & -379.26 & 0.76 & 4.87 & 1 & 0 \\
MOW & -387.56 & -379.02 & 0.15 & 0.83 & 0.09 & 0.203 \\
Weibull & -385.63 & -379.93 & 0.21 & 1.16 & 0.12 & 0.057 \\
GaW & -385.56 & -374.15 & 0.88 & 5.52 & 1 & 0 \\
KwW & -385.06 & -373.65 & 0.14 & 0.82 & 0.10 & 0.184 \\
McW & -382.97 & -368.71 & 0.14 & 0.80 & 0.83 & 0 \\
BW & -379.66 & -368.25 & 0.16 & 1.07 & 0.09 & 0.234 \\
\hline
\end{tabular}

Table 3: Estimated MLE's and Standard errors for the Phosphorus concentration in leaves data.

\begin{tabular}{l|cc}
\hline Model & MLE & Standard errors \\
\hline WTLG-W $(\alpha, \beta, \lambda, k)$ & $(15.80,0.25,0.12,1.90)$ & $(7.63,0.11,0.02,0.68)$ \\
ZBW $(\alpha, \lambda, k)$ & $(8.52,0.01,0.89)$ & $(0.92,0.002,0.05)$ \\
$\operatorname{MOW}(\alpha, \lambda, k)$ & $(0.27,0.20,3.59)$ & $(0.17,0.02,0.40)$ \\
Weibull $(\lambda, k)$ & $(0.16,2.82)$ & $(0.01,0.19)$ \\
$\operatorname{GaW}(\alpha, \beta, \lambda, k)$ & $(18.46,0.02,0.61,0.59)$ & $(29.62,0.01,1.26,0.48)$ \\
$\operatorname{KwW}(\alpha, \beta, \lambda, k)$ & $(8.01,6.35,0.09,0.75)$ & $(53.89,86.70,0.29,4.08)$ \\
$\operatorname{McW}(\alpha, \beta, c, \lambda, k)$ & $(3.45,10.69,8.73,0.03,0.41)$ & $(12.64,10.38,21.63,0.02,0.16)$ \\
$\operatorname{BW}(\alpha, \beta, \lambda, k)$ & $(77.74,1.12,0.01,0.58)$ & $(114.82,3.41,0.004,0.40)$ \\
\hline
\end{tabular}

Table 4: Hypothesis test result for the Phosphorus concentration in leaves data.

\begin{tabular}{l|ccc}
\hline Model & $-2 \log \Lambda \underset{\sim}{x})$ & P-Value & Hypothesis test result \\
\hline ZBW & 8.66 & 0.003 & null hypothesis is rejected \\
MOW & 8.89 & 0.003 & null hypothesis is rejected \\
Weibull & 12.84 & 0.005 & null hypothesis is rejected \\
\hline
\end{tabular}




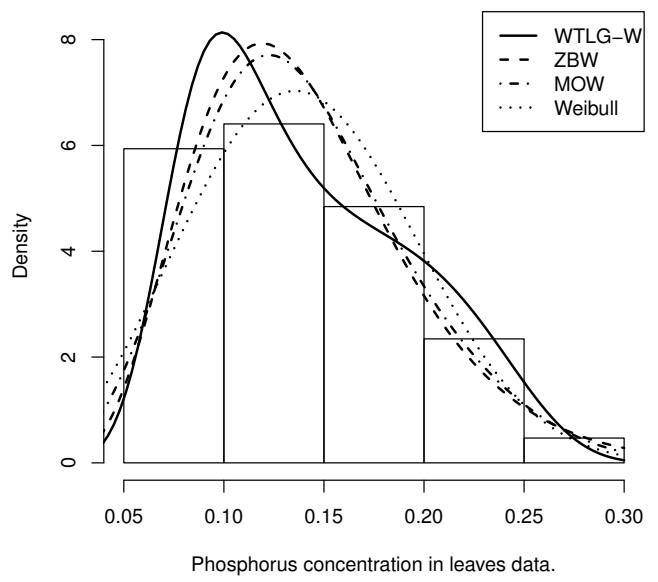

Figure 11: Histogram for Phosphorus concentration in leaves data set.

\subsection{Lifetimes of 50 Devices Data}

This sub-section is related to study of the lifetimes of 50 industrial devices put on life test at time zero. These data reported in Mudholkar and Srivastava (1993). The data set are the following:

$0.1,0.2,1,1,1,1,1,2,3,6,7,11,12,18,18,18,18,18,21,32,36,40,45,46,47,50,55,60$, $63,63,67,67,67,67,72,15,79,82,82,83,84,84,84,85,85,85,85,85,86,86$.

Similar to the previous application example, we have Tables 5 and 6. As it is clear, the WTLG-W is selected as the best model with more criteria (AIC, BIC, $W^{*}, A^{*}$ ). The histogram of the Lifetimes of 50 Devices data and the plots of fitted pdf are displayed in Figure 13. In Figure 14, the plot of uni-modality of profile likelihood functions of parameters of WTLG-W $(\alpha, \beta, \lambda, k)$ is shown.

As in the previous example, in Table 7, information about the hypothesis test is at $0.05 \%$ for test hypothesis in equation (6.1). As indicated in Table 7, the null hypothesis is rejected. 

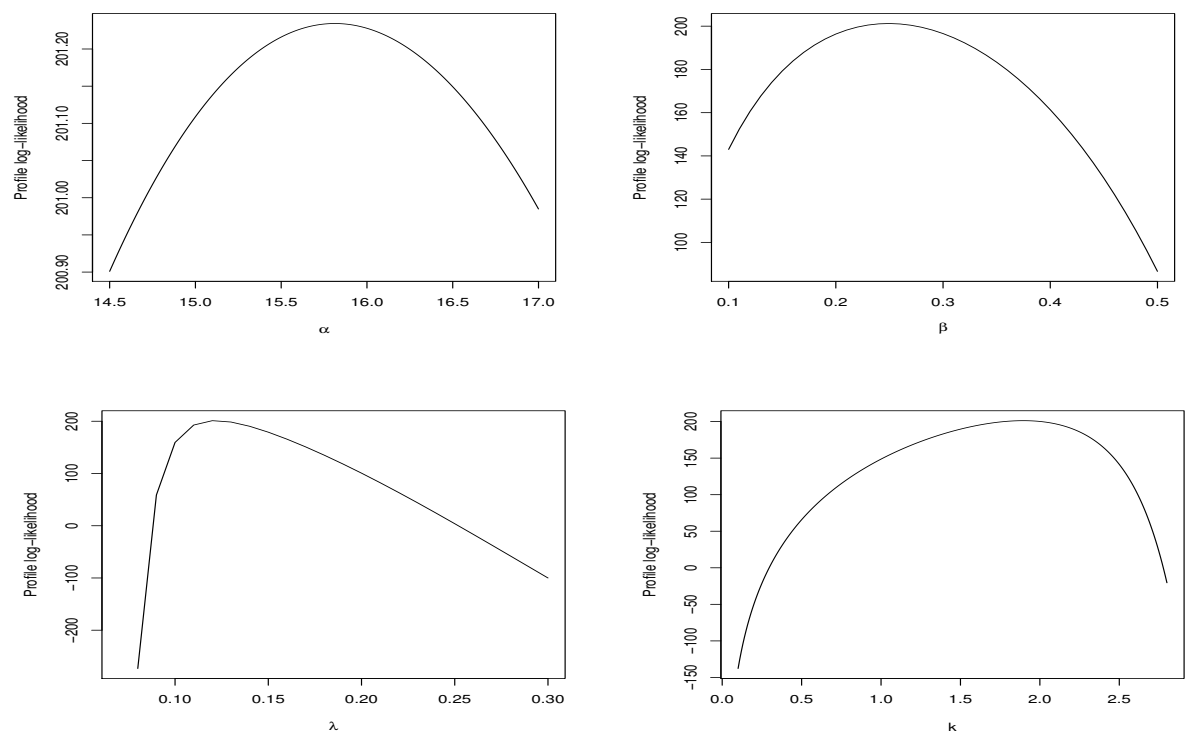

Figure 12: Uni-modality of profile likelihood functions of parameters of WTLG-W $(\alpha, \beta, \lambda, k)$ for Phosphorus concentration in leaves data set.

Table 5: Information criteria for the Lifetimes of 50 Devices data.

\begin{tabular}{l|cccccc}
\hline Model & AIC & BIC & $W^{*}$ & $A^{*}$ & K.S & P-Value \\
\hline WTLG-W & 439.91 & 447.56 & 0.09 & 0.76 & 0.16 & 0.158 \\
KwW & 451.84 & 459.48 & 0.15 & 1.18 & 0.14 & 0.283 \\
McW & 459.90 & 469.46 & 0.14 & 1.09 & 0.62 & 0 \\
ZBW & 462.41 & 468.15 & 0.60 & 3.54 & 0.87 & 0 \\
BW & 463.60 & 471.24 & 0.23 & 1.57 & 0.19 & 0.056 \\
MOW & 479.57 & 485.31 & 0.36 & 2.28 & 0.16 & 0.179 \\
Weibull & 483.25 & 487.07 & 0.43 & 2.70 & 0.18 & 0.068 \\
GaW & 508.67 & 516.32 & 1.42 & 7.28 & 1 & 0 \\
\hline
\end{tabular}


Table 6: Estimated MLE's and Standard errors for the Lifetimes of 50 Devices data.

\begin{tabular}{l|cc}
\hline Model & MLE & Standard errors \\
\hline WTLG-W $(\alpha, \beta, \lambda, k)$ & $(1.096,0.099,63.47,5.63)$ & $(0.2,0.01,0.76,0.003)$ \\
$\operatorname{KwW}(\alpha, \beta, \lambda, k)$ & $(0.06,0.26,59.09,5.10)$ & $(0.02,0.04,0.10,0.22)$ \\
$\operatorname{McW}(\alpha, \beta, c, \lambda, k)$ & $(0.02,39.40,7.51,96.24,5.04)$ & $(0.01,59.004,2.61,0.93,0.01)$ \\
ZBW $(\alpha, \lambda, k)$ & $(0.13,96.13,5.22)$ & $(0.02,0.61,0.003)$ \\
$\operatorname{BW}(\alpha, \beta, \lambda, k)$ & $(0.13,0.80,86.43,4.97)$ & $(0.02,0.23,1.45,0.01)$ \\
$\operatorname{MOW}(\alpha, \lambda, k)$ & $(6.07,13.96,0.69)$ & $(4.91,9.25,0.15)$ \\
Weibull $(\lambda, k)$ & $(43.47,0.94)$ & $(6.80,0.12)$ \\
$\operatorname{GaW}(\alpha, \beta, \lambda, k)$ & $(99.82,0.01,54.34,0.06)$ & $(5.58,0.001,27.51,0.01)$ \\
\hline
\end{tabular}

Table 7: Hypothesis test result for the Lifetimes of 50 Devices data.

\begin{tabular}{l|ccc}
\hline Model & $-2 \log \Lambda(\underset{\sim}{x})$ & P-Value & Hypothesis test result \\
\hline ZBW & 20.51 & $<0.00001$ & null hypothesis is rejected \\
MOW & 37.67 & $<0.00001$ & null hypothesis is rejected \\
Weibull & 43.35 & $<0.00001$ & null hypothesis is rejected \\
\hline
\end{tabular}

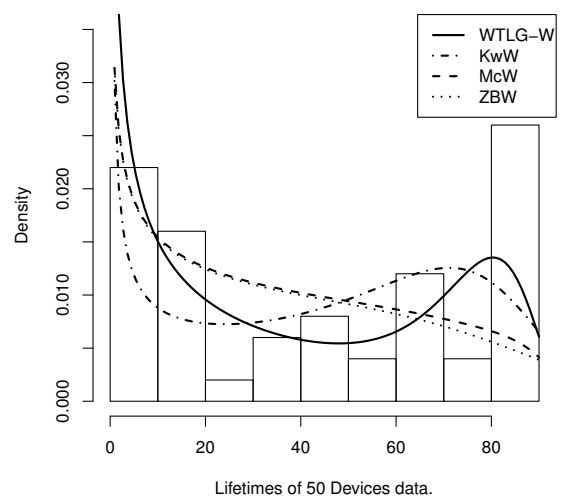

Figure 13: Histogram for the Lifetimes of 50 Devices data. 

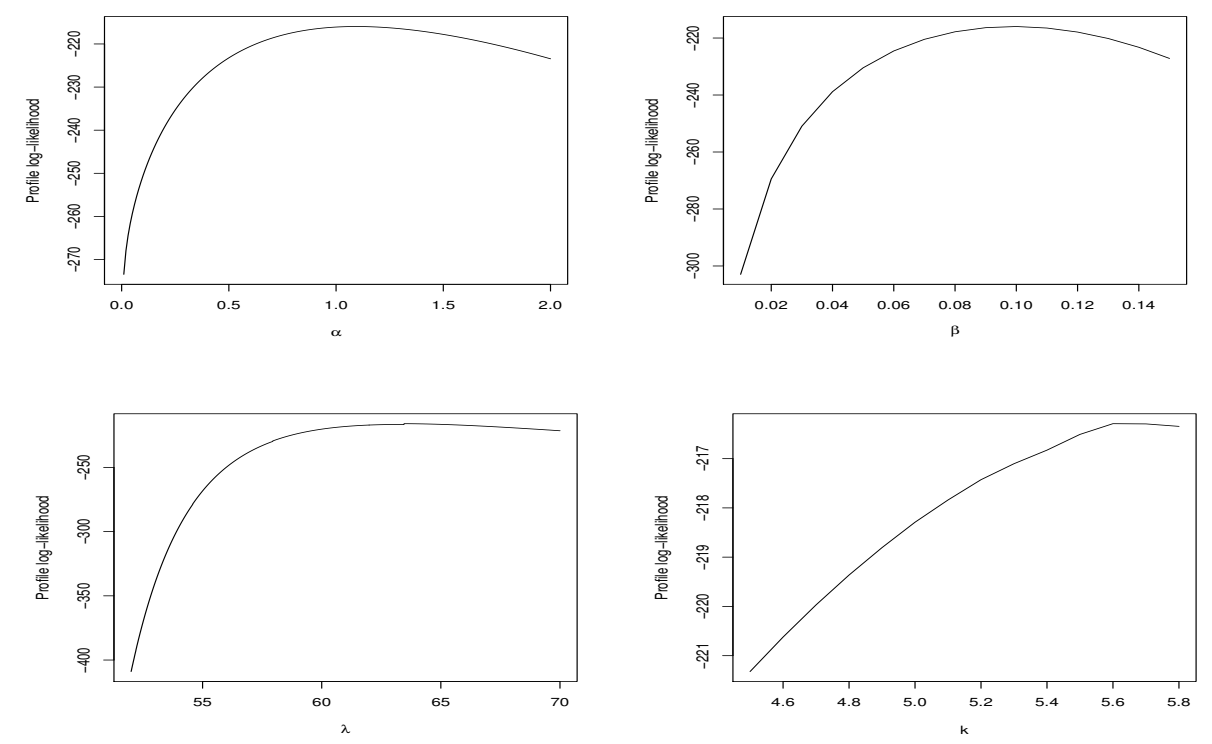

Figure 14: Uni-modality of profile likelihood functions of parameters of WTLG-W $(\alpha, \beta, \lambda, k)$ for the Lifetimes of 50 Devices data.

\section{Concluding Remarks}

In this paper, we introduce a new class of lifetime distributions called the Weibull ToppLeone Generated (WTLG) family. Some of its mathematical properties are derived. The maximum likelihood method and five other methods are used for estimating the parameters. The Bias and MSE of the estimators for all methods will approach zero with the increase in the volume of the sample which verifies the validity of these estimation methods. The likelihood ratio test illustrates the goodness of the WTLG family. The WTLG is applied to fit two real data sets. It can provide better fits than some of the known lifetime distributions. The importance and flexibility of the new family are illustrated via applications.

The results of tables and figures have shown the usefulness of the WTLG family for applied statistical research. $R$ codes are available in the appendix. 


\section{References}

Afify, A. Z., Yousof, H. M. and Nadarajah, S. (2017), The beta transmuted-H family of distributions: properties and applications. Stasistics and its Inference, 10, 505-520.

Alizadeh, M., Ghosh, I., Yousof, H. M., Rasekhi, M. and Hamedani G. G. (2017), The generalized odd generalized exponential family of distributions: properties, characterizations and applications. Journal of Data Science, 16, 443-446.

Alizadeh, M., Korkmaz, M. C., Almamy, J. A. and Ahmed, A. A. E. (2018), Another odd log-logistic logarithmic class of continuous distributions. Journal of Statisticians: Statistics and Actuarial Sciences, 11(2), 55-72.

Anderson, T. W. and Darling, D. A. (1952), Asymptotic theory of certain" goodness of fit" criteria based on stochastic processes. The annals of mathematical statistics, 193-212.

Alzaatreh, A., Lee, C. and Famoye, F. (2013), A new method for generating families of continuous distributions. Metron, 71, 63-79.

Alzaatreh, A., Famoye, F. and Lee, C. (2014), The gamma-normal distribution: Properties and applications. Computational Statistics and Data Analysis, 69, 67-80.

Brito, E., Cordeiro, G. M., Yousof, H. M., Alizadeh, M. and Silva , G. O. (2017), ToppLeone odd log-logistic family of distribution. Journal of Statistical Computation and Simulation, 87(15), 3040-3058.

Bourguignon, Silva M., R. B. and Cordeiro, G. M. (2014), The Weibull-G Family of Probability Distributions. Journal of Data Science, 12, 53-68.

Choi, K. and Bulgren, W. (1968), An estimation procedure for mix- tures of distributions. Journal of the Royal Statistical Society. Series B (Methodological), 444-460.

Cooray, K. (2006), Generalization of the Weibull distribution: the odd Weibull family. Statistical Modelling, 6, 265-277.

Cooray, K. and Ananda, M. M. (2008), A generalization of the half-normal distribution with applications to lifetime data. Communications in Statistics-Theory and Methods, 37(9), 1323-1337.

Cordeiro, G. M., Ortega, E. M. M. and Nadarajah, S. (2010), The Kumaraswamy Weibull distribution with application to failure data. Journal of the Franklin Institute, 347, 13991429. 
Cordeiro, G. M., Ortega, E. M. and da Cunha, D. C. C. (2013), The exponentiated generalized class of distributions. Journal of Data Science, 11, 1-27.

Dey. S., Mazucheli, J. and Nadarajah.S. (2017), Kumaraswamy distribution: different methods of estimation. Computational and Applied Mathematics, 1-18.

Eugene, N., Lee, C., and Famoye, F. (2002), Beta-normal distribution and its applications. Communications in Statistics - Theory and Methods, 31, 497-512.

Famoye, F., Lee, C. and Olumolade, O. (2005), The beta-Weibull distribution. Journal of Statistical Theory and Applications, 4(2), 121-136.

Fonseca, M. B. and Franca, M. G. C. (2007), A influoencia da fertilidade do solo e caracterizacao da fixacao biologica de N2 para o crescimento de Dimorphandra wilsonii rizz. Master thesis, Universidade Federal de Minas Gerais.

Glänzell, W. (1990), Some consequences of a characterization theorem based on truncated moments. Statistics, 21(4), 613-618.

Gupta, R. D., and Kundu, D. (2001), Exponentiated exponential family: an alternative to gamma and Weibull. Biometrical Journal, 43, 117-130.

Gupta, R. C., Gupta, P. L., and Gupta, R. D. (1998), Modeling failure time data by Lehmann alternatives.Communications in Statistics - Theory and Methods, 27, 887-904.

Hamedani, G. G. (2013), On certain generalized gamma convolution distributions II (No. 484). Technical Report.

Hamedani, G. G., Altun, E., Korkmaz, M. C., Yousof, H. M., and Butt, N. S. (2018), A new extended $G$ family of continuous distributions with mathematical properties, characterizations and regression modeling. Pakistan Journal of Statistics and Operation Research, 14(3), 737-758.

Hashimoto, E. M, Ortega, E. M. M., Cordeiro, G. M., and Pascoa, M. A. R. (2015), The McDonald Extended Weibull Distribution. Journal of Statistical Theory and Practice, 9(3), 608-632.

Korkmaz, M. C., and Genc, A. I. (2017), A new generalized two-sided class of distributions with an emphasis on two- sided generalized normal distribution. Communications in Statistics-Simulation and Computation, 46(2), 1441-1460. 
Korkmaz, M. C., Yousof, H. M., and Hamedani, G. G. (2018a), The Exponential Lindley Odd Log-Logistic-G Family: Properties, Characterizations and Applications. Journal of Statistical Theory and Applications, 17(3), 554-571.

Korkmaz, M. C., Yousof H. M., Hamedani, G. G., and Ali M. M. (2018b), The MarshallOlkin Generalized G Poisson Family Of Distributions. Pakistan Journal of Statistics, $34(3), 251-267$.

Korkmaz, M. C., Alizadeh, M., Yousof, H. M., and Butt, N. S. (2018c), The generalized odd Weibull generated family of distributions: statistical properties and applications. Pakistan Journal of Statistics and Operation Research, 14(3), 541-556.

Korkmaz, M. C. (2019a), A new family of the continuous distributions: the extended Weibull-G family. Communications Faculty of Sciences University of Ankara Series A1 Mathematics and Statistics 68(1), 248-270.

Korkmaz, M. C., Cordeiro, G. M., Yousof, H. M., Pescim, R. R., Afify, A. Z., and Nadarajah, S. (2019b), The Weibull Marshall-Olkin family: Regression model and application to censored data. Communications in Statistics-Theory and Methods Accepted, DOI: 10.1080/03610926.2018.1490430.

Lindley, D. V. (1958), Fiducial distributions and Bayes' theorem. Journal of the Royal Statistical Society, Series B, 20, 102-107.

Marshall, A. W., and Olkin, I. (1997), A new methods for adding a parameter to a family of distributions with application to the Exponential and Weibull families. Biometrika, 84, 641-652.

Mudholkar, G. S., and Srivastava, D. K. (1993), Exponentiated Weibull family for analysing bathtub failure rate data. IEEE Transactions on Reliability, 42, 299-302.

Nadarajah, S., Cordeiro, G. M., and Ortega, E. M. M., (2014), The ZografosBalakrishnan-G Family of Distributions: Mathematical Properties and Applications. Communications in Statistics - Theory and Methods, 44, 186-215.

Nofal, Z. M., Afify, A. Z., Yousof, H. M., and Cordeiro, G. M. (2017), The generalized transmuted-G family of distributions. Communications in Statistics - Theory and Methods, 46, 4119-4136.

Pogany, T. K., Saboor, A., and Provost, S., (2015), The Marshall Olkin Exponential Weibull Distribution. Hacettepe Journal of Mathematics and Statistics, 44(6), 1579-1594. 
Silva, R. B., Bourguignon, M., Dias, C. R. B., and Cordeiro, G. M. (2013), The compound class of extended Weibull power series distributions. Computational Statistics and Data Analysis, 58, 352-367.

Swain, J. J., Venkatraman, S., and Wilson, J. R., (1988), Least- squares estimation of distribution functions in johnson's translation system. Journal of Statistical Computation and Simulation, 29, 271- 297.

Yousof, H. M., Afify, A. Z., Alizadeh, M., Butt, N. S., Hamedani, G. G., and Ali, M. M. (2015), The transmuted exponentiated generalized-G family of distributions. Pakistan Journal of Statistics and Operation Research, 11, 441-464.

Yousof, H. M., Afify, A. Z., Hamedani, G. G., and Aryal, G. (2016), the Burr X generator of distributions for lifetime data. Journal of Statistical Theory and Applications, 16, 288-305.

Yousof, H. M., Majumder, M., Jahanshahi, S. M. A., Ali, M. M., and Hamedani, G. G. (2018), A new Weibull class of distributions: theory, characterizations, and applications. Journal of Statistical Research of Iran, 23, 13-31. 


\section{Appendix A: $R$ code}

The program is developed in $\mathrm{R}$ to obtain the value of CDF function of WTLG-W distribution:

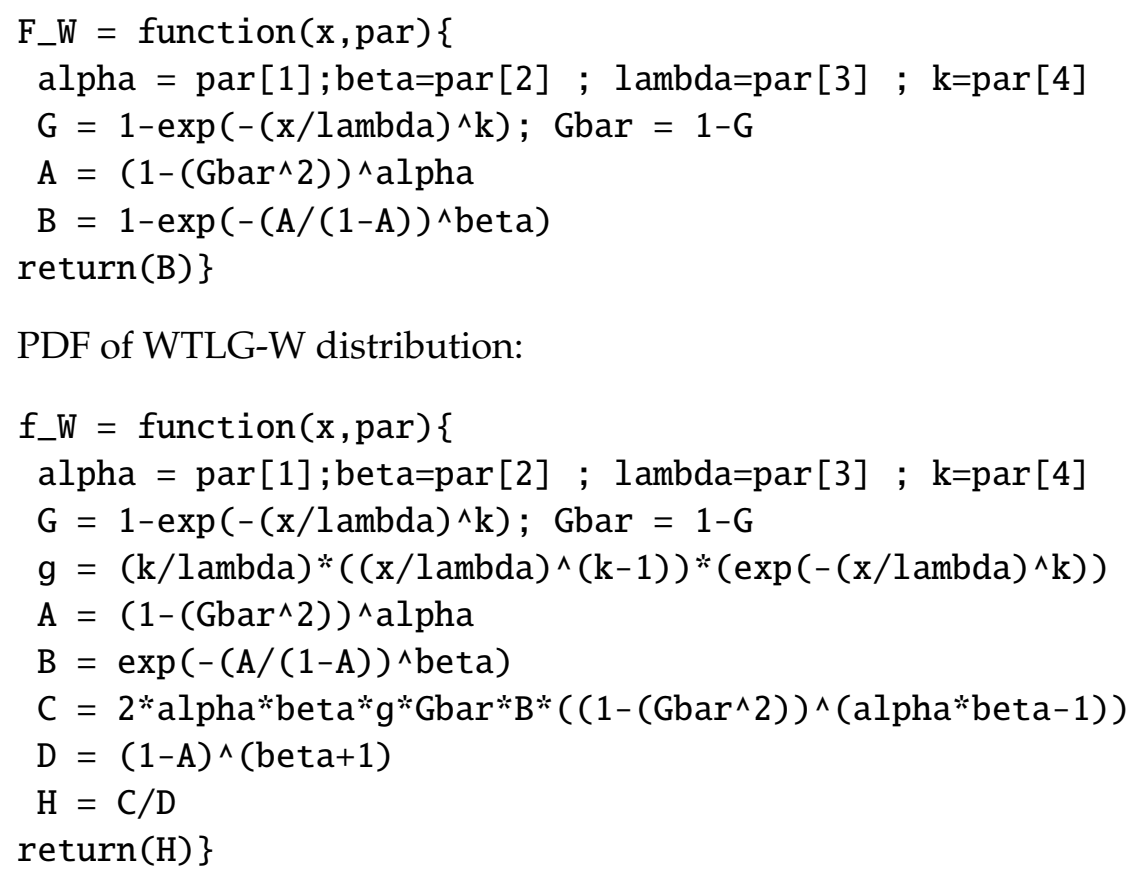

CDF of WTLG-N distribution:

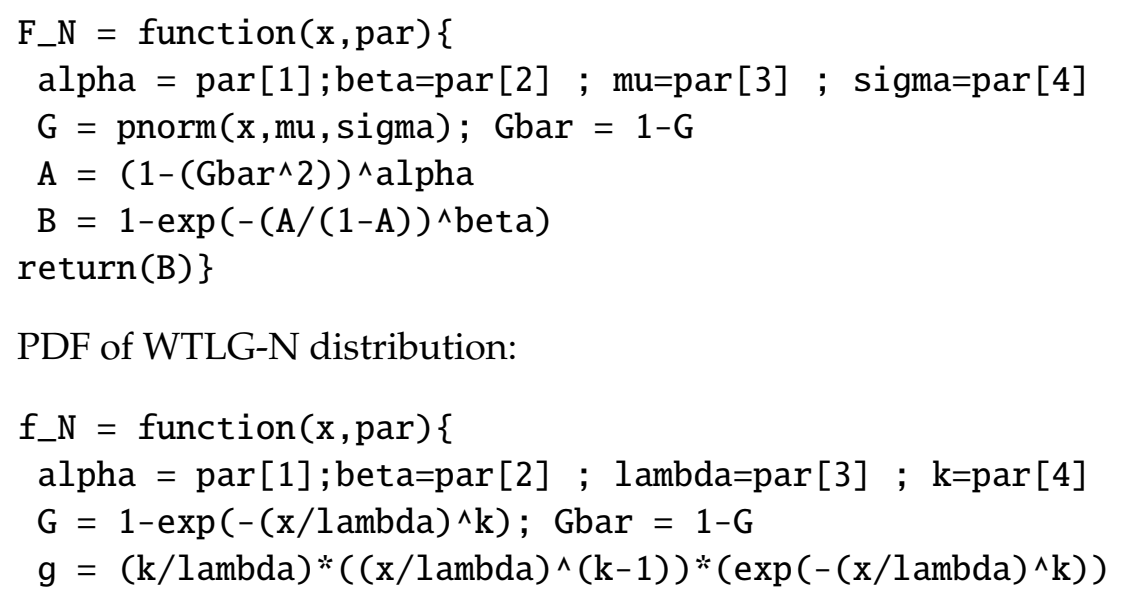




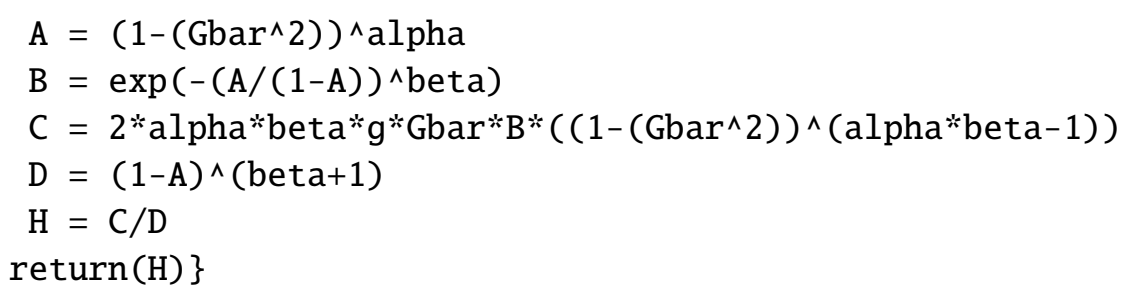

Hazard function:

$h=$ function $(x$, par $) f(x, p a r) /(1-F(x, p a r))$

The program is developed in $\mathrm{R}$ to obtain the value of skewness (kurtosis):

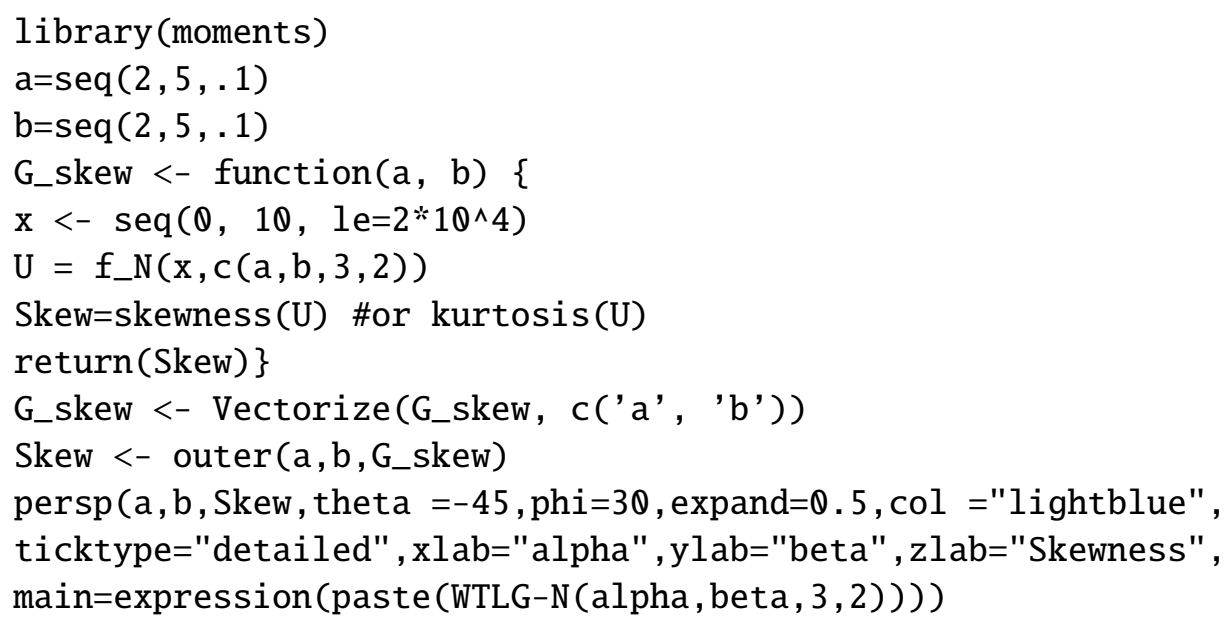

The program is developed in $\mathrm{R}$ to generate of WTLG-W distribution (for example WTLG-W $(2,2,3,2))$ :

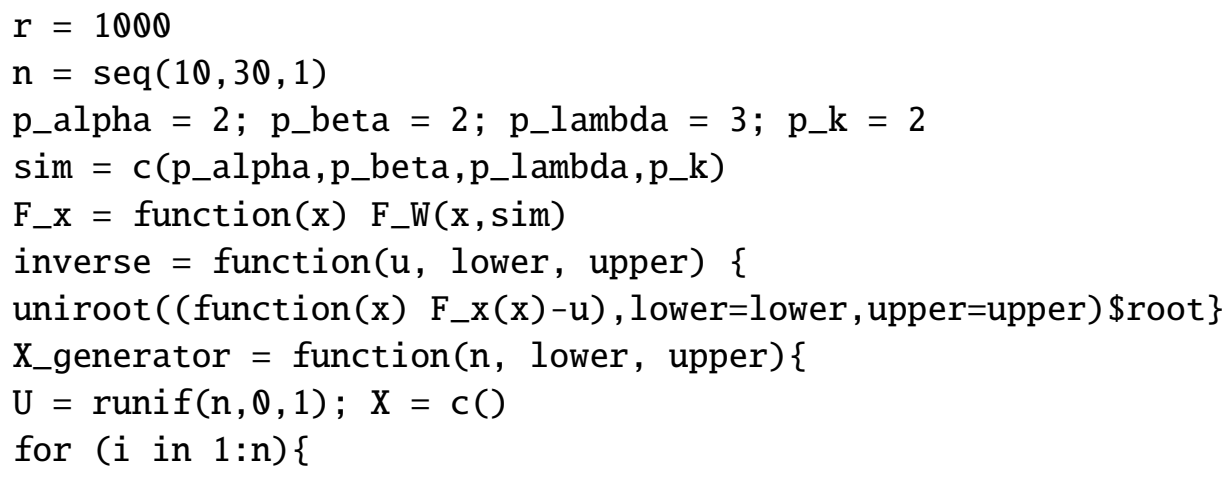


$X[i]=i n v e r s e(U[i]$, lower, upper) $\}$

$\operatorname{return}(\mathrm{X})\}$

The program is developed in $\mathrm{R}$ to obtain the value of likelihood function:

LikFun_f $=$ function $(x, p a r)\{$

alpha=par [1]; beta=par [2]; lambda=par [3];k=par [4]

$\mathrm{G}=1-\exp \left(-(\mathrm{x} / \mathrm{lambda})^{\wedge} \mathrm{k}\right) ; \quad$ Gbar $=1-\mathrm{G}$

$\mathrm{g}=(\mathrm{k} / \mathrm{l} \text { ambda })^{*}\left((\mathrm{x} / \mathrm{lambda})^{\wedge}(\mathrm{k}-1)\right)^{*}\left(\exp \left(-(\mathrm{x} / \mathrm{l} \text { ambda })^{\wedge} \mathrm{k}\right)\right)$

$A=\left(1-\left(\operatorname{Gbar}^{\wedge} 2\right)\right)^{\wedge}$ alpha

$B=\exp \left(-(A /(1-A))^{\wedge}\right.$ beta $)$

$C=2 * a l p h a *$ beta*g*Gbar*B*((1-(Gbar^2))^(alpha*beta-1))

$\mathrm{D}=(1-\mathrm{A})^{\wedge}($ bet $\mathrm{a}+1)$

$\mathrm{H}=\operatorname{prod}(\mathrm{C} / \mathrm{D})$

$\operatorname{return}(\mathrm{H})\}$

The program is developed in $\mathrm{R}$ to compute the value of Bias and MSE with $\mathrm{r}$ iterate and sample size $\mathrm{n}$ (for example for $\alpha$ parameter):

for $(\mathrm{k}$ in $1:$ length $(\mathrm{n}))\{$

for $(j$ in $1: r)\{$

$\mathrm{X}=\mathrm{X}$ _generator $(\mathrm{n}[\mathrm{k}], 0,500)$

MLE $=$ optim(par $=$ sim, $f$ n=LikFun_f, $x=X)$

alpha_mle $[j]=$ MLE\$par[1]

bias_alpha1[j] = alpha_mle[j]-p_alpha

MSE1_alpha[j]=(bias_alpha1[j] $\left.)^{\wedge} 2\right\}$

bias_alpha[k]= mean(bias_alpha1)

MSE_alpha $[\mathrm{k}]=$ mean(MSE1_alpha)\}

\section{Appendix B}

Theorem 1. Let $(\Omega, \mathcal{F}, \mathbf{P})$ be a given probability space and let $H=[a, b]$ be an interval for some $d<b \quad(a=-\infty, b=\infty$ might as well be allowed). Let $X: \Omega \rightarrow H$ be a continuous random variable with the distribution function $F$ and let $q_{1}$ and $q_{2}$ be two real functions defined on $H$ such that

$$
\mathbf{E}\left[q_{2}(X) \mid X \geq x\right]=\mathbf{E}\left[q_{1}(X) \mid X \geq x\right] \xi(x), \quad x \in H,
$$


is defined with some real function $\eta$. Assume that $q_{1}, q_{2} \in C^{1}(H), \xi \in C^{2}(H)$ and $F$ is twice continuously differentiable and strictly monotone function on the set $H$. Finally, assume that the equation $\xi q_{1}=q_{2}$ has no real solution in the interior of $H$. Then $F$ is uniquely determined by the functions $q_{1}, q_{2}$ and $\xi$, particularly

$$
F(x)=\int_{a}^{x} C\left|\frac{\xi^{\prime}(u)}{\xi(u) q_{1}(u)-q_{2}(u)}\right| \exp (-s(u)) d u,
$$

where the function $s$ is a solution of the differential equation $s^{\prime}=\frac{\xi^{\prime} q_{1}}{\xi q_{1}-q_{2}}$ and $C$ is the normalization constant, such that $\int_{H} d F=1$.

We like to mention that this kind of characterization based on the ratio of truncated moments is stable in the sense of weak convergence (see, Glänzel [2]), in particular, let us assume that there is a sequence $\left\{X_{n}\right\}$ of random variables with distribution functions $\left\{F_{n}\right\}$ such that the functions $q_{1 n}, q_{2 n}$ and $\xi_{n}(n \in \mathbb{N})$ satisfy the conditions of Theorem 1 and let $q_{1 n} \rightarrow q_{1}, q_{2 n} \rightarrow q_{2}$ for some continuously differentiable real functions $q_{1}$ and $q_{2}$. Let, finally, $X$ be a random variable with distribution $F$. Under the condition that $q_{1 n}(X)$ and $q_{2 n}(X)$ are uniformly integrable and the family $\left\{F_{n}\right\}$ is relatively compact, the sequence $X_{n}$ converges to $X$ in distribution if and only if $\xi_{n}$ converges to $\xi$, where

$$
\xi(x)=\frac{E\left[q_{2}(X) \mid X \geq x\right]}{E\left[q_{1}(X) \mid X \geq x\right]} .
$$

This stability theorem makes sure that the convergence of distribution functions is reflected by corresponding convergence of the functions $q_{1}, q_{2}$ and $\xi$, respectively. It guarantees, for instance, the 'convergence' of characterization of the Wald distribution to that of the Lévy-Smirnov distribution if $\alpha \rightarrow \infty$.

A further consequence of the stability property of Theorem 1 is the application of this theorem to special tasks in statistical practice such as the estimation of the parameters of discrete distributions. For such purpose, the functions $q_{1}, q_{2}$ and, specially, $\xi$ should be as simple as possible. Since the function triplet is not uniquely determined it is often possible to choose $\xi$ as a linear function. Therefore, it is worth analyzing some special cases which help to find new characterizations reflecting the relationship between individual continuous univariate distributions and appropriate in other areas of statistics. 
In some cases, one can take $q_{1}(x) \equiv 1$, which reduces the condition of Theorem 1 to $\mathbf{E}\left[q_{2}(X) \mid X \geq x\right]=\xi(x), \quad x \in H$. We, however, believe that employing three functions $q_{1}, q_{2}$ and $\xi$ will enhance the domain of applicability of Theorem 1. 